doi:10.1016/j.fct.2005.11.001

Copyright (c) 2006 Elsevier Ltd All rights reserved.

\title{
Probabilistic dietary exposure assessment taking into account variability in both amount and frequency of consumption
}

\section{Wout Slob ${ }^{a_{1}}$ *}

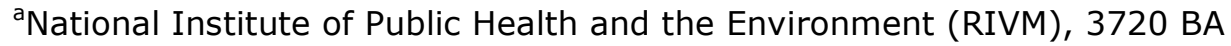

Bilthoven, The Netherlands

* Tel.: +3130 2743242; fax: +31302744475.

\begin{abstract}
Probabilistic dietary exposure assessments that are fully based on Monte Carlo sampling from the raw intake data may not be appropriate. This paper shows that the data should first be analysed by using a statistical model that is able to take the various dimensions of food consumption patterns into account. A (parametric) model is discussed that takes into account the interindividual variation in (daily) consumption frequencies, as well as in amounts consumed. Further, the model can be used to include covariates, such as age, sex, or other individual attributes. Some illustrative examples show how this model may be used to estimate the probability of exceeding an (acute or chronic) exposure limit. These results are compared with the results based on directly counting the fraction of observed intakes exceeding the limit value. This comparison shows that the latter method is not adequate, in particular for the acute exposure situation. A two-step approach for probabilistic (acute) exposure assessment is proposed: first analyse the consumption data by a (parametric) statistical model as discussed in this paper, and then use Monte Carlo techniques for combining the variation in concentrations with the variation in consumption (by sampling from the statistical model). This approach results in an estimate of the fraction of the population as a function of the fraction of days at which the exposure limit is exceeded by the individual.
\end{abstract}

\section{Introduction}

Human intakes of compounds present in foods are often estimated using consumption data from food consumptions surveys. In these surveys a large sample of individuals have provided a detailed account of their food consumption on a given day, either by keeping a diary, or by responding to an interview the day after (recall method). For most purposes, interest is not only in the average consumption behavior of humans, but also in its variability. For instance, for the purpose of risk assessment of a contaminant occurring in food, knowledge of the intake of the average (median) individual would not be sufficient: the goal normally is to protect a large fraction of the population rather than just half of it. When the average intake is not too far away from the exposure limit, variability in food consumption should therefore be taken into account in the exposure assessment. 
Variability in daily consumptions is complicated. First of all, individuals differ from each other in their (long-term average) consumption habits. Further, individuals show different consumption behaviors on different days. An extra complicating factor is that the number of different food items that people may consume is very large. While some food items are consumed regularly, others are consumed only incidentally. Hence, consumption has two aspects that need to be taken into account: the amount of a particular product that is consumed, but also the selection of products that are consumed on a single day. Accordingly, modeling dietary exposure should consider both of these aspects, i.e., not only how much of a food product is being consumed, but also how often.

Although food consumption surveys provide consumption information for a number of days in each individual, this number of days is limited, and far from sufficient to provide an accurate estimate of the long-term consumption behavior in each particular individual. The food consumption surveys do however provide information to estimate the distribution of consumption behavior among individuals. Several papers have discussed methods or estimating the distribution of consumption amounts (e.g., Slob, 1993a, Slob, 1993b and Nusser et al., 1996). The methods discussed in these papers work well as long as (virtually) all observed daily intakes are nonzero. In many situations, however, a large part of the observed intakes is zero. These are often referred to by the term "nonconsumers". This is unfortunate terminology, since an individual that scores zero intakes on all observed days may be a consumer on the next (or any other) unobserved day. To distinguish people in just two categories, consumers and nonconsumers, is totally inadequate. For most foods there are frequent consumers, less frequent consumers, incidental consumers, etc., and dichotomization is highly unrealistic. Rather, each individual has its own consumption frequency (= probability of nonzero consumption on a single day), an entity that varies between individuals, just as the (average) amount consumed typically varies between individuals. To arrive at a realistic description of the intake in the population, it is therefore necessary to describe consumption frequencies as a distribution as well. This means that an exposure model should not only include a distribution for the amount consumed, but also for the consumption frequency.

The practical question that arises is whether such a distribution can at all be estimated from food consumption survey data, where individuals have been followed only for a couple of days, in some surveys even as few as two (as in the Dutch surveys). Intuitively, this appears unlikely. After all, there is not much information in two days where an individual was observed not to consume any apples, regarding the true consumption frequency of apples in that individual. Nonetheless, this paper shows some results from computer simulations illustrating that this is yet possible, if a particular type of distribution for the consumption frequencies is assumed, and if the sample size of observed individuals is large enough. To maintain a sufficiently large sample size of individuals, it is important to describe consumption frequency as a function of age, rather than discriminating different age classes (e.g., years of age) and perform the analysis separately for each age class. That approach would lead to sample sizes that are too small for estimating the consumption frequency distribution.

Next to estimating the consumption frequency distribution, the distribution of the amounts consumed (given a consumption occasion) should also be estimated. This distribution reflects both variation between individuals and within individuals (between days), and both these components of variation can be estimated as described earlier (e.g., Slob, 1993a and Nusser et al., 1996). 
Food and Chemical Toxicology Volume 44, Issue 7 , July 2006, Pages 933-951

This paper discusses a statistical model for describing consumption patterns of single food products in the human population, by combining both the variability in consumption frequency and in amounts consumed. The model is fully parametric, while other (semiparametric) approaches have been proposed by others (Nusser et al., 1996 and Dodd, 1996). Some of the reasons for choosing a full parametric model will be discussed below.

Once the model has been fitted to the intake data, it can be assessed which fraction of the population would have a long-term (usual) intake exceeding a given exposure limit (e.g., the Reference Dose, RfD, or the Acceptable Daily Intake, ADI). Also, it can be calculated which fraction of the population would exceed an acute exposure limit (Acute RfD) how often ( = what proportion of days). This indirect approach of estimating these fractions from the fitted model will be compared to the direct approach of estimating them from the observations directly. These comparisons will illustrate that the direct method may be quite inaccurate. The conclusion of this paper is that probabilistic exposure assessments that are based on Monte Carlo sampling from the consumption data themselves are inadequate for dietary intakes. Instead, it is proposed to apply Monte Carlo sampling from the statistical model of consumption behaviour, and combine this approach with Monte Carlo sampling from the concentration data.

\section{General description of the model}

The model discussed here (coined STEM.II: Statistical Exposure Model for Incidental Intakes) describes the consumption of a single food product in the whole population, taking account of variation between individuals regarding the amounts as well as the frequency of consumption of the product. For the purpose of dietary exposure assessment it needs to be combined with information on concentrations in foods. How this may be done is discussed at the end of the paper.

Consumption frequency is defined as the fraction (in statistical terms, the expected fraction) of days that the food product is consumed. It is assumed that individuals vary in consumption frequency, some showing a very low frequency, some a very high frequency, and other persons somewhere in between.

Therefore, consumption frequency may be assumed to follow some (unknown) statistical distribution. It will be assumed that the consumption frequency can be described by the beta-distribution. This distribution is restricted to the domain $(0,1)$, in accordance to consumption frequency being restricted to this range. It has two parameters, $a$ and $\beta$, with the expected (mean) consumption frequency being equal to $a /(a+\beta)$. It is shown below (Section 4$)$ that this distribution can be reasonably estimated when the total number of daily intakes is in the order of 10,000 . For much smaller sample sizes the estimates are not reliable however. Therefore, splitting the data up in different age classes (e.g., years of age), and analysing these separately, would result in too small sample sizes. To avoid this, the (mean) consumption frequency may be described by a regression function of age, thereby assuming that one of the parameters $a$ and $\beta$ remains constant. Here, parameter $a$ is chosen as the constant parameter (taking parameter $\beta$ constant resulted in similar estimates, results not shown here). In this way the complete data set of intakes for all ages can be used to estimate the betadistribution (i.e., the constant parameter $a$ ) around the mean consumption frequency, which itself is estimated as a regression function of age (see Fig. 3 for an illustration).

Consumption amounts are described as a function of age as well, with a scatter around this function that contains both the variation between and within 
individuals. Both these components of variation are assumed to be log-normally distributed in fitting the model to the data. This assumption may (and should) be checked afterwards, and other transformations could be applied, if necessary.

It may be noted that the approach of describing consumption frequencies as a function of age assuming parameter $a$ to be constant is analogous to that of fitting a regression function to the intake amounts, assuming that the variation around the mean is constant over age. Another analogy is that for both frequencies and amounts the regression analysis can be easily extended to examine certain covariates, such as sex, social class, or ethnic background, by assuming that particular regression parameters depend on the level of the covariate. For instance, it might be that the function of age is similar for both sexes, but that the overall level differs (resulting in two parallel curves). This situation may be expressed by assuming parameter a (see below) to depend on sex, refit the model and check if the increase in the number of parameters in the model resulted in a significantly better fit (using the likelihood ratio test). If it did, the covariate may be included into the regression model. In this way, more efficient use is made of the information in the data compared to the situation where the males and females were analysed separately. This approach is illustrated below.

After having decided which regression function will be used for describing the intake amounts (see Fig. 7 for an illustration), the regression residuals are plotted in a QQ-plot (see Fig. 9 for an illustration) to check if the lognormal distribution gives a reasonable description on intake amounts, and to examine possible outliers. Finally, the observed variation of the regression residuals is corrected for the variation within individuals (daily fluctuations), resulting in an estimate of the variation in usual intakes among individuals.

For some products there might be a correlation between (usual) amounts consumed by individuals and their consumption frequencies. The food consumption surveys contain information on the existence of such a correlation, and this can be examined by plotting the amounts consumed as a function of observed fraction of days with nonzero consumption of the relevant individuals (for an illustration, see Fig. 5). It is however hardly possible to quantify this relationship such that it can be included in the model. For instance, with only two observed days per individual, the observed relationship does not represent the real relationship. Here, individuals with two observed consumption days in reality represent individuals with all sorts of mean consumption frequencies, possibly even including values below 0.5 . For the same reason, the scatter around the observed mean amounts does not really represent the variation at a single consumption frequency. Therefore, the observed amounts as a function of observed consumption frequency, as well as the variation around the mean amounts give a biased view of the true relationship. Of course, the situation improves with increasing number of observed days per individual, but even then it does not seem easy to derive the relationship accurately.

\section{Statistical description of the model}

\subsection{Consumption frequency}

The expected consumption frequency, $E n$, is assumed to be a function of age, $t$ :

$$
E n=f\left(\theta_{n} ; t\right)
$$


where $\theta_{\Pi}$ denotes the vector of regression parameters of the function $f$. The variable $n$ is assumed to follow a beta-distribution with parameters $a$ and $\beta$. Parameter $a$ is assumed constant over age, so that $\beta$ is a function of age as well, since $E n=a /(a+\beta)$. This model is fitted to the (zero or nonzero) daily intakes by Maximum Likelihood based on the beta-binomial distribution (see e.g., Teunis and Slob, 1999), where parameter $a$ is estimated simultaneously with the regression parameters $\theta_{\Pi}$.

The assumption that $a$ is constant over age may be checked for any particular dataset as follows. Subdivide the total age span into a number of bins, and assume that $a$ is only constant within these bins. Then refit the same model, and compare the obtained log-likelihood value with the one obtained earlier for the model with a single value of $a$ over the whole age span. These log-likelihood values may be compared by the log-likelihood ratio test, to see if the model with heterogeneous $a$ results in a significant improvement of the fit. If not, the assumption of homogenous $a$ is not rejected by the data.

\subsection{Intake amounts}

Intake amounts (on nonzero consumption days), $Y_{i j}(t)$, are described by

$$
\log \left[Y_{i j}(t)\right]=g\left(\theta_{Y} ; t\right)+\varepsilon_{i}+\delta_{i j}
$$

where $Y_{i j}(t)$ is the intake amount (absolute or relative to body weight) of individual $i$, having age $t$, on day $j, g\left(\theta_{Y} ; t\right)$ is the typical (usual) log-intake amount of the typical individual at age $t, \varepsilon_{i}$ is the deviation of individual $i$ 's typical log-intake amount compared to that of the typical individual and $\delta_{i j}$ is the deviation of individual $i$ 's log-intake amount on day $j$ compared to that person's typical log-intake.

It is assumed that the variances of $\varepsilon_{i}$ and $\delta_{i j}$ (between-subject and within-subject variances, respectively) are both homogeneous. Furthermore, it is assumed that $\varepsilon_{i}$ and $\delta_{i j}$ have normal distributions with zero means. It follows that the backtransformation of $g\left(\theta_{Y} ; t\right)$ reflects the median individual in the population. More generally, for any transformation of the intakes resulting in a normal (or at least symmetric) distribution the back-transformed $g\left(\theta_{Y} ; t\right)$ reflects the median individual in the population. Further, the typical (usual) intake on consumption days in individual $i$ is the median $\left(M_{i}\right)$ of (nonzero) daily intakes in that individual, which is lognormally distributed as

$$
M_{i}(t)-\Lambda\left(g(t) ; \sigma_{u}^{2}\right)
$$

while the intake on a single day is lognormally distributed as

$$
Y_{i j} \sim \Lambda\left(M_{i}(t) ; \sigma_{i}^{2}\right)
$$

Note that the usual (median) intake in any individual is a function of age as well, and that these functions of age are assumed to be parallel among individuals (on log-scale).

Of course, the log-transformation in expression (1) can be replaced by another transformation if the data would ask for that. 
The vector $\theta_{Y}$ of regression parameters is estimated by fitting the function $g$ to the observed daily intake amounts plotted as a function of age. The regression residuals are subjected to a nested ANOVA, resulting in an estimate of the variance within individuals $\left(\sigma_{\tilde{b}}^{2}\right)$. This value is subtracted from the total observed variance, to achieve the variance between individuals $\left(\sigma_{s}^{2}\right)$. Alternatively, the between variance is often estimated based on the expected value of the MS between. Although both methods should normally result in the same result, the latter estimate sometimes results in negative estimates of the between variance.

\subsection{Correlation between consumption frequency and amount}

Although a relationship between usual intake and consumption frequency theoretically could be included in the model, it is not trivial to estimate this relationship (including the variation around it) from food consumption survey data, in particular for surveys with only two days (as in the Dutch surveys). Therefore, this issue will not be further discussed in this paper, except for checking if the assumption that the relationship can be ignored. If the relationship is not negligible, this should be reported in the final results, to indicate that the final results may be subject to some bias, which may be conservative (when the correlation is negative) or anti-conservative (when the correlation is positive).

\subsection{Non-monotone regression functions}

The regression functions $f$ (for expected consumption frequency) and $g$ (for median intake amounts) may be chosen to be any function that is able to describe the data as a function of age. A useful nested family of functions is given by

$$
\begin{aligned}
y(t)= & a\left\{\left[c_{1}-\left(c_{1}-1\right) \exp \left(\frac{-t}{b_{1}}\right)^{d_{1}}\right]\right. \\
& \left.\times\left[c_{2}-\left(c_{2}-1\right) \exp \left(\frac{-t}{b_{2}}\right)^{d_{2}}\right]\right\},
\end{aligned}
$$

since it is very flexible, and includes both monotone and non-monotone functions. Which member of this family should be used for describing a particular data set may be decided by using the maximum likelihood ratio as a criterion (see, e.g., Slob, 2002).

\subsection{Regression of consumption frequencies}

The function just mentioned can also be applied to consumption frequencies, which is particularly useful when the consumption frequencies show a nonmonotone relationship with age. This may be done by assuming that the regression function holds for a latent variable $y$, which is a continuous variable supposed to follow a lognormal distribution. This continuous latent variable is translated into a binary variable by assuming some cut-off value (th1 in the figure legends, where th1 is on log-scale) in the underlying lognormal distribution below which the binary value is zero, and unity otherwise. The parameter th 1 is estimated in fitting the model to the data. The parameters a (value of $y$ at age $=0$ ) and sig (standard deviation of the underlying normal distribution) are both fixed to unity (to prevent over-parameterization). This approach is similar to categorical regression as applied to ordinal data (e.g., Hertzberg, 1989), but with 
just one category of effect, and with more flexibility regarding the dose-response function for the latent variable $y$.

\section{Computer simulations}

As already noted, food survey data do not provide sufficient information on the long-term consumption behavior of each particular individual in the survey. This holds for the average amount consumed of a given product, but even more so for the consumption frequency of that individual. For instance, the observation of two zero consumption days in the same person is far from sufficient to estimate that person's personal consumption frequency. In surveys with more than two observation days per individual the situation improves somewhat, but even then the individual estimates are poor. What matters is, however, whether or not the variation (distribution) of consumption frequency in the whole population can be estimated, using food survey data. To explore this some computer simulations were performed. In these simulations, a particular beta-distribution was assumed, representing the true distribution of consumption frequencies. From that distribution a number of values (persons) were randomly drawn, and based on each sampled value a binomial sample was drawn for each individual, representing a realization of the number of consumption or non-consumption days in that individual.

Fig. $1 \mathrm{~A}-\mathrm{C}$ shows the results for three different beta-distributions, comparing study designs that have the same total number $(12,000)$ of observed intakes, with different number of observation days per individual. In general, the median estimates of the simulation results are quite close to the true value for the median and 95th percentile of the beta-distribution for all designs. Only in the third distribution (with lowest mean frequency) the median of the distribution is overestimated, while the 95th percentile is underestimated. Increasing the number of days per individual decreases the bias, but with more then 10 days the precision of these estimates decreases at the same time. In the second distribution (Fig. 1B) it is found that parameters $a$ and $\beta$ may be substantially overestimated, while the median and 95th percentile are estimated quite accurately. This may be explained by the strong correlation between the estimates of $a$ and $\beta$. Overall, the optimal number of days appears to lie somewhere between 5 and 10 days according to these simulations. 
Food and Chemical Toxicology Volume 44, Issue 7 , July 2006, Pages 933-951
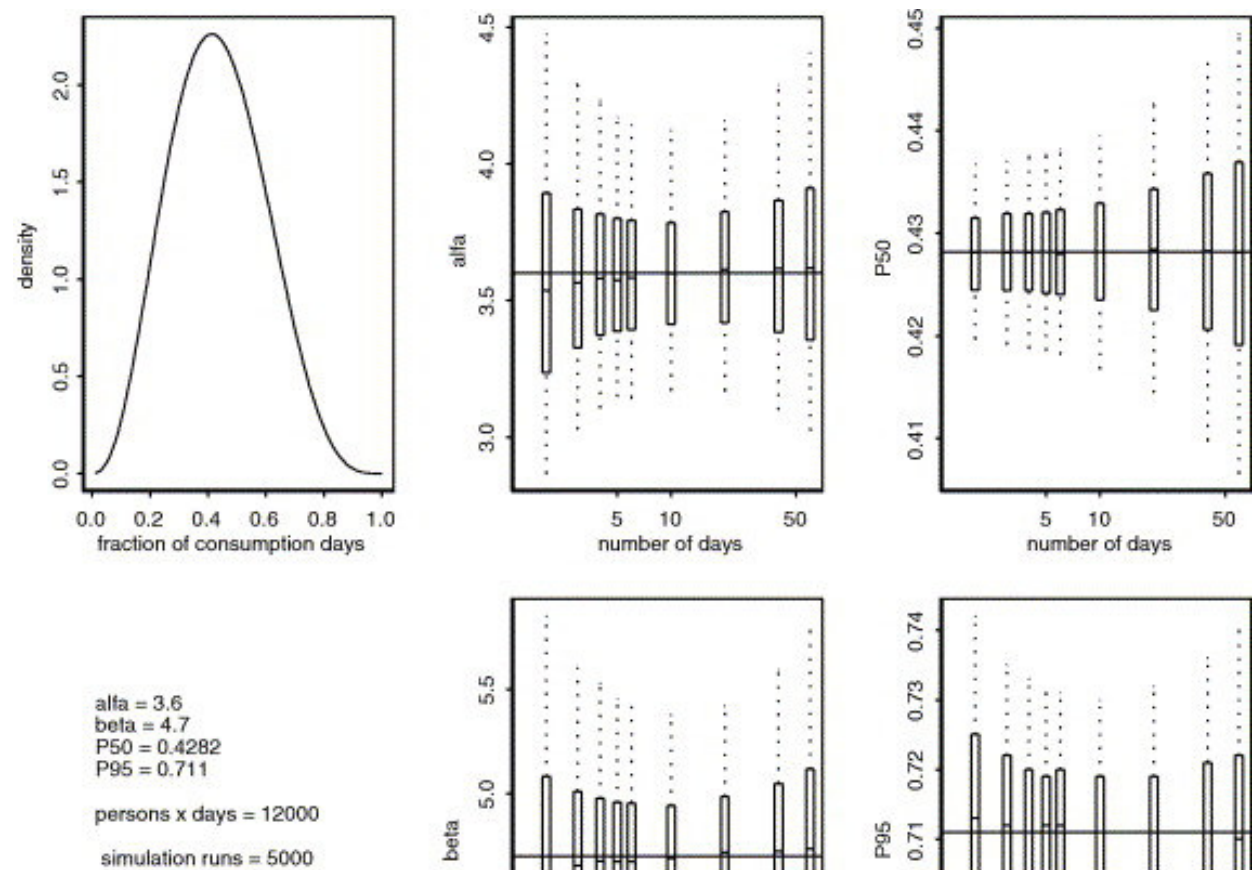

(A)
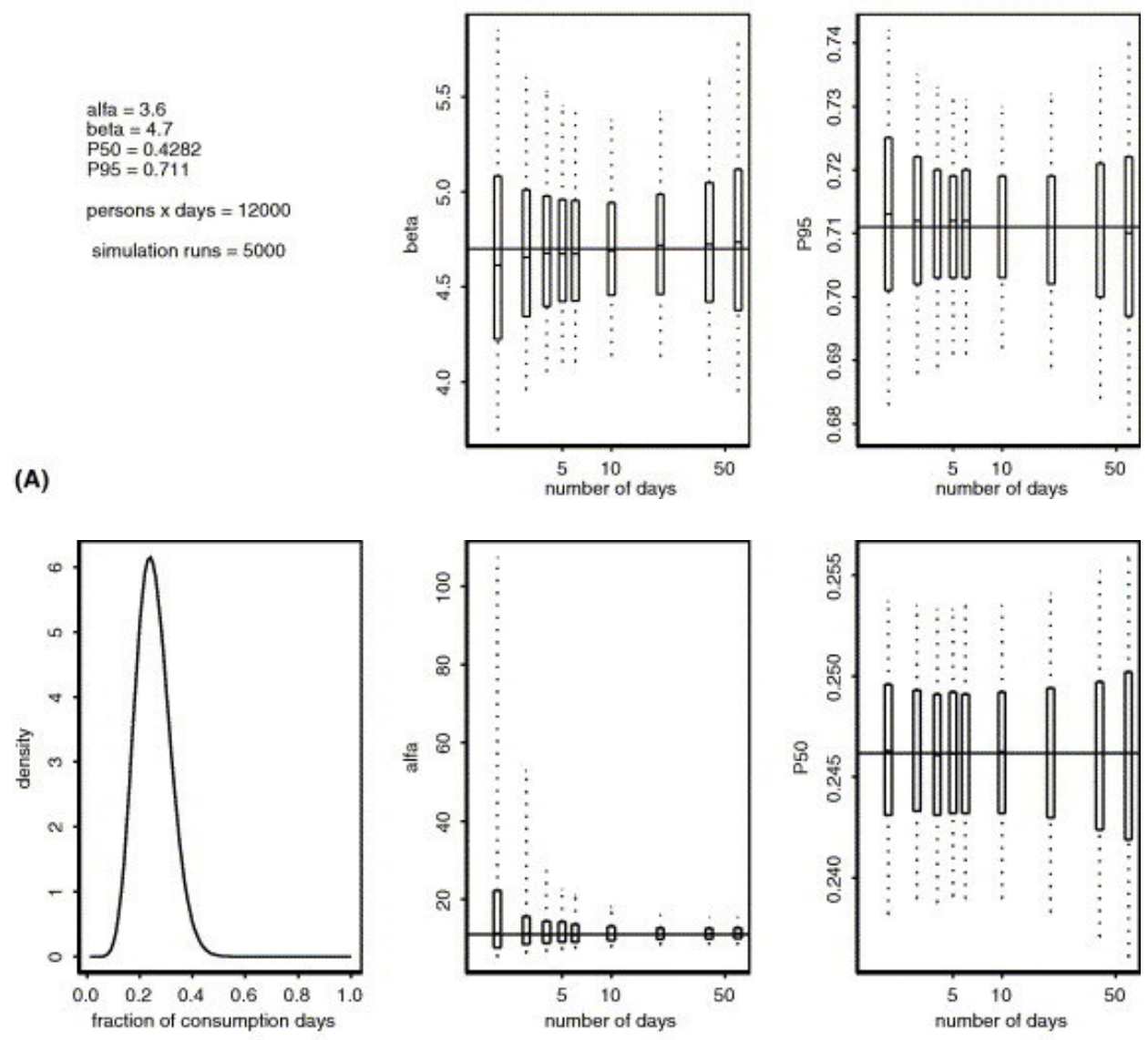

alfa $=11$

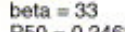

$P 50=0.2462$

$\mathrm{P} 95=0.362$

persons $x$ days $=12000$

simulation runs $=5000$

(B)
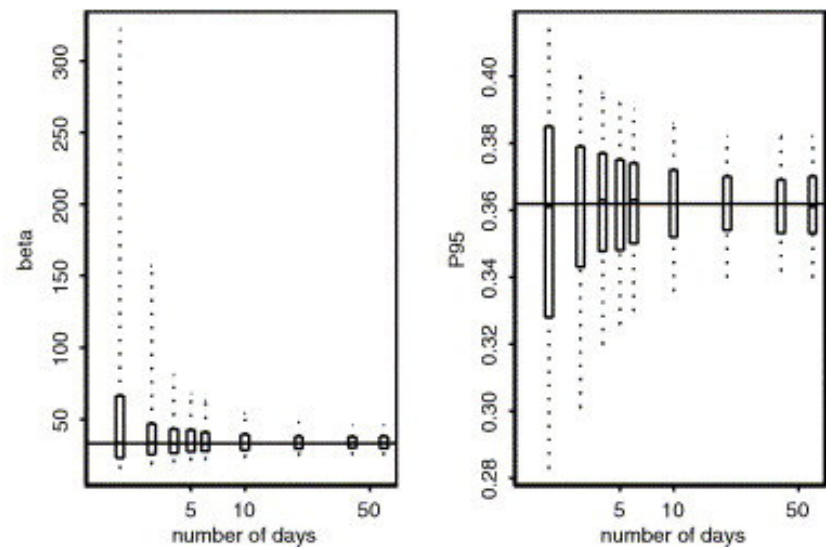

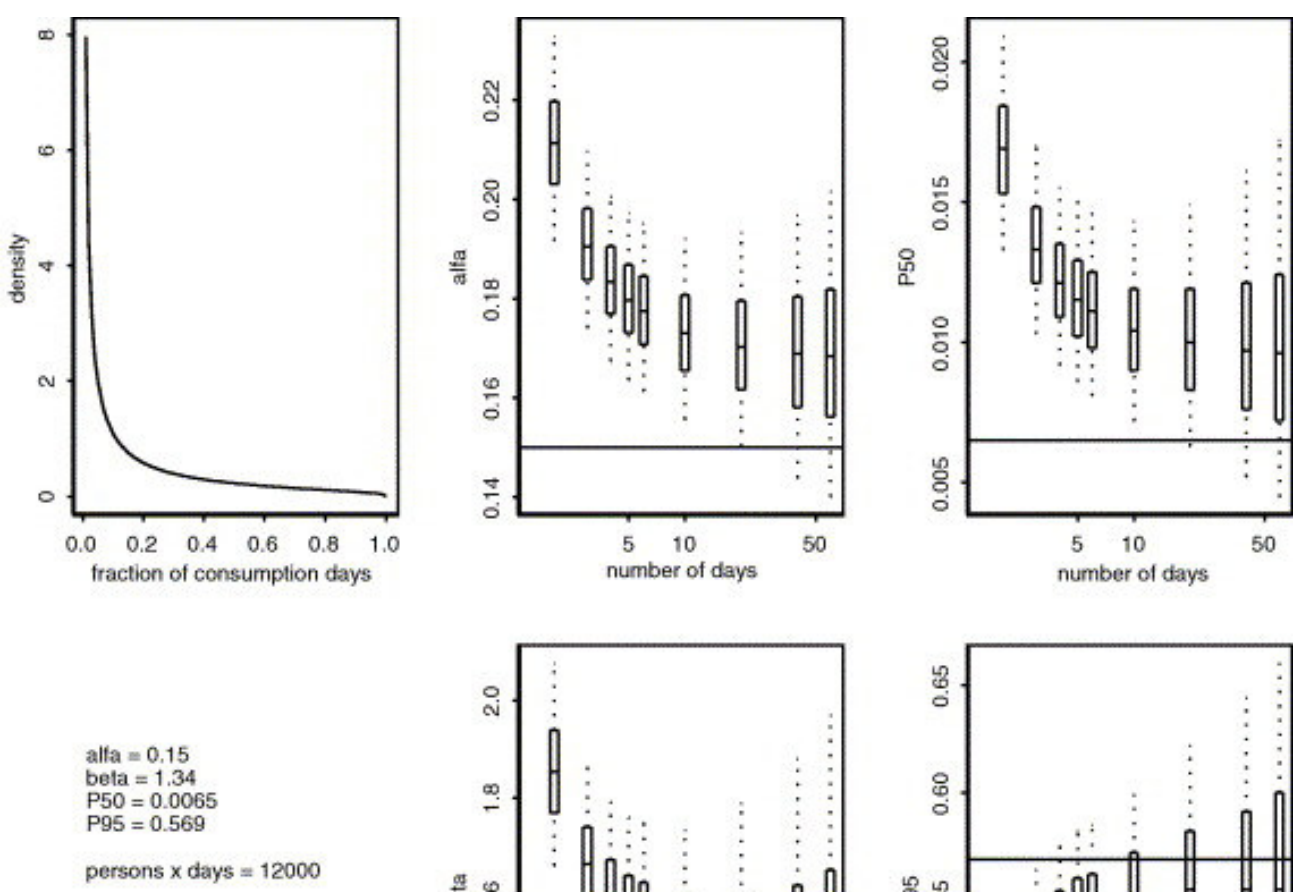

simulation runs $=5000$

(C)
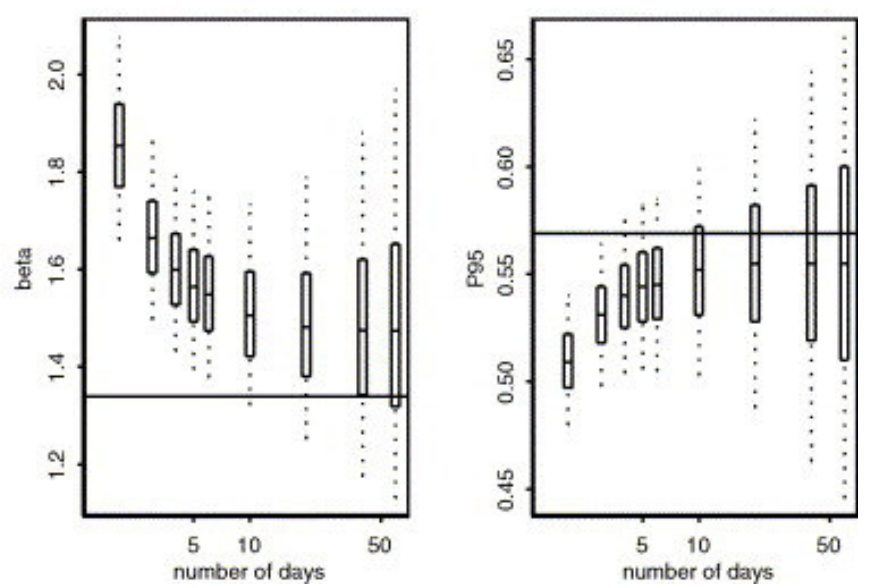

Fig. 1. Simulation results for estimating a beta-distribution as plotted in the upper left panel (see lower left panel for some characteristics), by varying the number of days per individual and keeping the total number of observations at 12,000. The four panels on the right show box-plots for the simulation results (5th, 25th, 50th, 75th and 95th percentiles), for alfa, beta, the median (P50) and the 95th percentile $(P 95)$. The horizontal lines denote the true values.

Obviously, decreasing the number of persons (for a given number of days per person) will result in poorer estimates of the beta-distribution. As an illustration, Fig. 2 shows some simulation results (for the same distribution as in Fig. 1C) to get an impression of how fast the precision decreases with smaller surveys. For instance, a survey with 100 persons and five registration days resulted in simulation estimates of the distribution's 95th percentile somewhere between 0.3 and 0.75 , which implies that such a design would be inadequate for the purpose of estimating the consumption frequency distribution. This also implies that analyzing the observations for each separate age class is no option. 

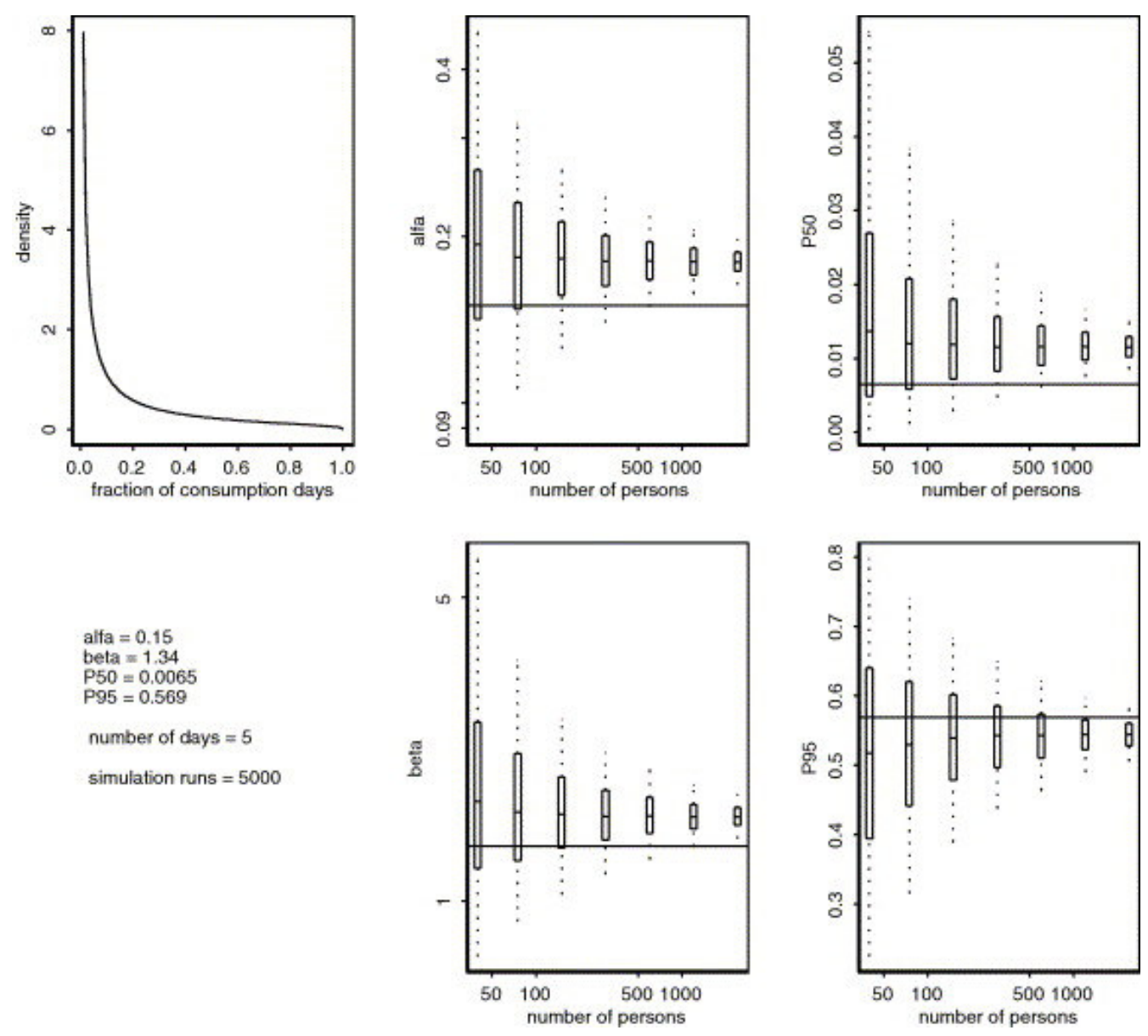

Fig. 2. Simulation results for estimating a beta-distribution as plotted in the upper left panel (see lower left panel for some characteristics), by varying the number of individuals (40,75, 150,300,600, 1200 and 2400, plotted on log-scale) and keeping the number of days at 5 . The four panels on the right show box-plots for the simulation results (5th, 25th, 50th, 75th and 95th percentiles), for alfa, beta, the median $(P 50)$ and the 95th percentile $(P 95)$. The horizontal lines denote the true values.

\section{Example analyses}

This section illustrates the application of the model to some example food products, based on the Dutch food consumption survey that was carried out in the year 1997/1998 (Anon., 1998).

\subsection{Analysis of consumption frequency}

Fig. 3 shows the (average) fraction of observed consumption days for potatoes plotted as a function of age. A regression function is fitted to the fractions as observed in each individual, to describe the relationship of (mean) consumption frequency with age. In fitting the regression function, the parameter $a$ of the beta-distribution is estimated at the same time. The resulting beta-distribution at age 40 is plotted in the same figure, to indicate the variation in consumption frequency among individuals (at that age). 


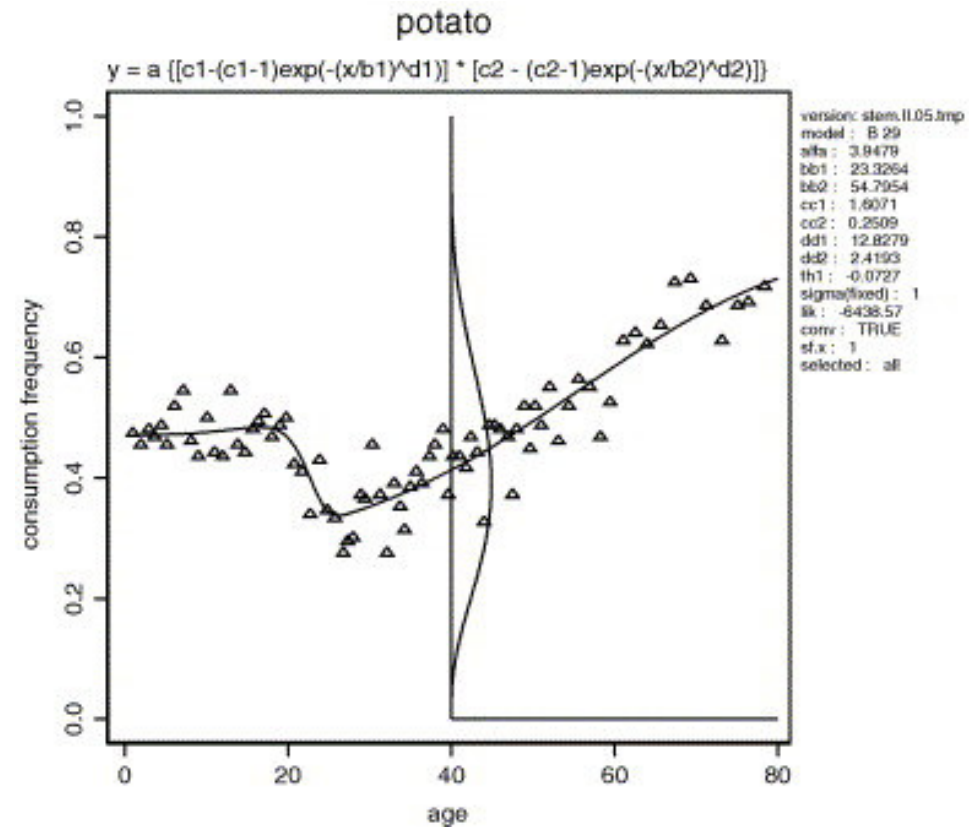

Fig. 3. Observed (average) consumption frequencies for potatoes, plotted against years of age, together with a fitted regression model. Note that the model was fitted to the individual binomial data ( 0,1 or 2 out of 2 days), not to the average frequencies, although the latter are plotted here. The parameter $a$ (alfa in the legend) of the beta-distribution was estimated simultaneously in fitting the model. The resulting beta-distribution for age class 40 years is plotted. For explanation of parameters th1 and sigma see main text (Section 3).

In this case, the relationship of consumption frequency with age is clearly nonmonotone. The regression function that was fitted (see top of figure) has the flexibility to follow such non-monotone relationships.

The same model was refitted after allowing parameter a being different between sexes. This did not result in a significantly better fit, actually the two curves virtually overlapped. This result indicates that males and females do not really differ in potato consumption frequency.

As another example, Fig. 4 shows the consumption frequency of wine. The scatter of the observed frequencies is larger in this plot, which is related to the (average) frequencies being lower than in the potato case. Here, the fit of the regression model did significantly improve by assuming the parameter $a$ to be different between sexes. As a result, two curves are plotted, one for each sex, the curve for females being higher than the one for males. Actually, a separate analysis of males and females resulted in an even (significantly) higher log-likelihood value in this case, the shapes of the regression functions being different between males and females (results not shown). 


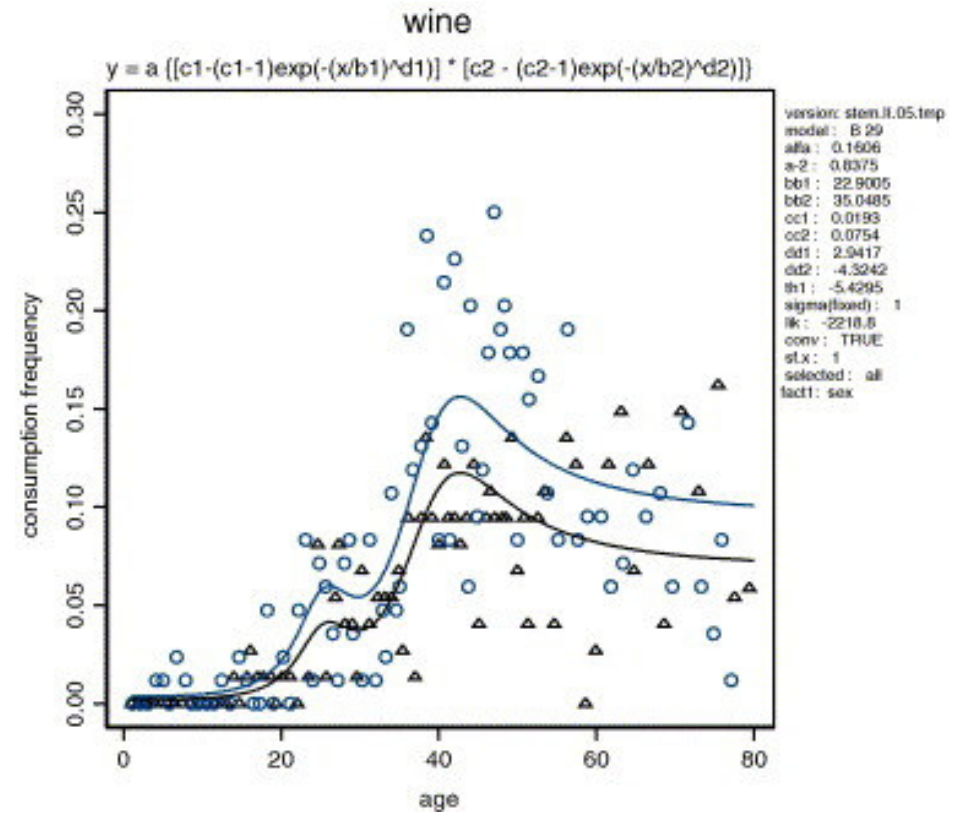

Fig. 4. Observed (average) consumption frequencies of wine (triangles for males, circles for females) plotted against years of age, together with a fitted regression model (upper curve relates to females). Here, the regression model was fitted while regression parameter a was allowed to depend on age. The value of a was fixed at 1 for males, while for females it was estimated at 0.838 (indicated by $a-2$ in the legend). This fit was significantly better than the fit with parameter $a$ being equal for both sexes. The parameter $a$ (alfa in the legend) of the betadistribution was estimated simultaneously in fitting the model. For explanation of parameters th1 and sigma see main text (Section 3 ).

More generally, other covariates than sex, such as social class, ethnic background can all be efficiently examined in the same way as illustrated here for the covariate sex. Any of such potential differences will hardly be detectable in an analysis of separate age classes.

The assumption that parameter $a$ (one of the parameters of the beta-distribution) is constant over age can be checked by including various parameters $a$ into the model, each associated with a particular age range. Table 1 shows some results for five different food products. In all cases, including a single parameter $a$ (i.e., including a beta-distribution with constant $a$ ) leads to a dramatic increase in the log-likelihood value. Adding more parameters $a$ did not lead to a statistically significant improvement of the fit, except for potato, where increasing the number of parameters $a$ from one to five did result in a significant improvement of the fit ( $P=0.02$, likelihood ratio test). However, to obtain a significantly better fit it appears to be sufficient to assume just two parameters $a$, one for children, and one or adults, as the associated fit of this model was by itself significantly better than the model with constant $a(P=0.006)$, while it was not improved by the model with five parameters $a$. 
Table 1.

Log-likelihood values for various model fits, including various numbers of parameters $a$ in the model

\begin{tabular}{|l|l|l|l|l|l|}
\hline Number of parameters $\boldsymbol{a}$ & Wine & Potato & Pear & Grapes & Eggs \\
\hline None & -2760.26 & -8316.91 & -2369.91 & -1269.06 & -7362.10 \\
\hline 1 & -2218.80 & -6438.57 & -1941.51 & -1055.65 & -5657.78 \\
\hline 2 (Ages: 1-18, 19-80) & & $-6434.75^{\mathrm{a}}$ & & & \\
\hline 5 & -2218.11 & -6432.56 & -1937.47 & -1053.97 & -5654.93 \\
\hline 10 & -2216.96 & -6430.25 & -1934.11 & -1050.58 & -5653.71 \\
\hline
\end{tabular}

For models with more than one $a$, the age range is subdivided in equidistant bins. For potato, a special case is considered, where the age intervals 1-18 and 19-80 are assumed to differ in $a$.

a Significant improvement compared to model one row higher.

These results indicate that the assumption of constant $a$ appears quite reasonable in general. To some extent this may also be considered as indirect evidence that the beta-distribution adequately describes consumption frequencies in the human population for many food products. Had the beta-distribution been highly unrealistic, this error in distribution would probably have been corrected for in the model fit by estimating different values for $a$.

\subsection{Analysis of consumption amount}

The Dutch food consumption survey contains only two registration days per individual, and a potential relationship between consumption amount and consumption frequency can only be assessed qualitatively, as already noted. As an example, Fig. 5 shows the observed amounts of wine consumed for persons that had consumed wine on both, or on just one of the two days. In this case, there seems to be a positive correlation between amount and frequency of consumption, more frequent wine consumers drinking on average more per day. The ratio of the two geometric means was 1.21. However, this estimate is not completely reliable, since effects of other covariates, such as age, may interfere. Therefore, it is better to first perform a regression analysis of amounts with age, and possibly other covariates, as discussed below. But even then it should be noticed that the observed fraction of consumption days ( $x$-axis in Fig. 5 ) does not reflect the true consumption frequencies of the individuals in the long run. For instance, the individuals that happened to have used wine on the two registration days most certainly do no (exclusively) comprise individuals that drink wine every day of the year. For that reason, it is hard to know the true (quantitative) relationship between amount consumed per day and (long-term) consumption frequency. 

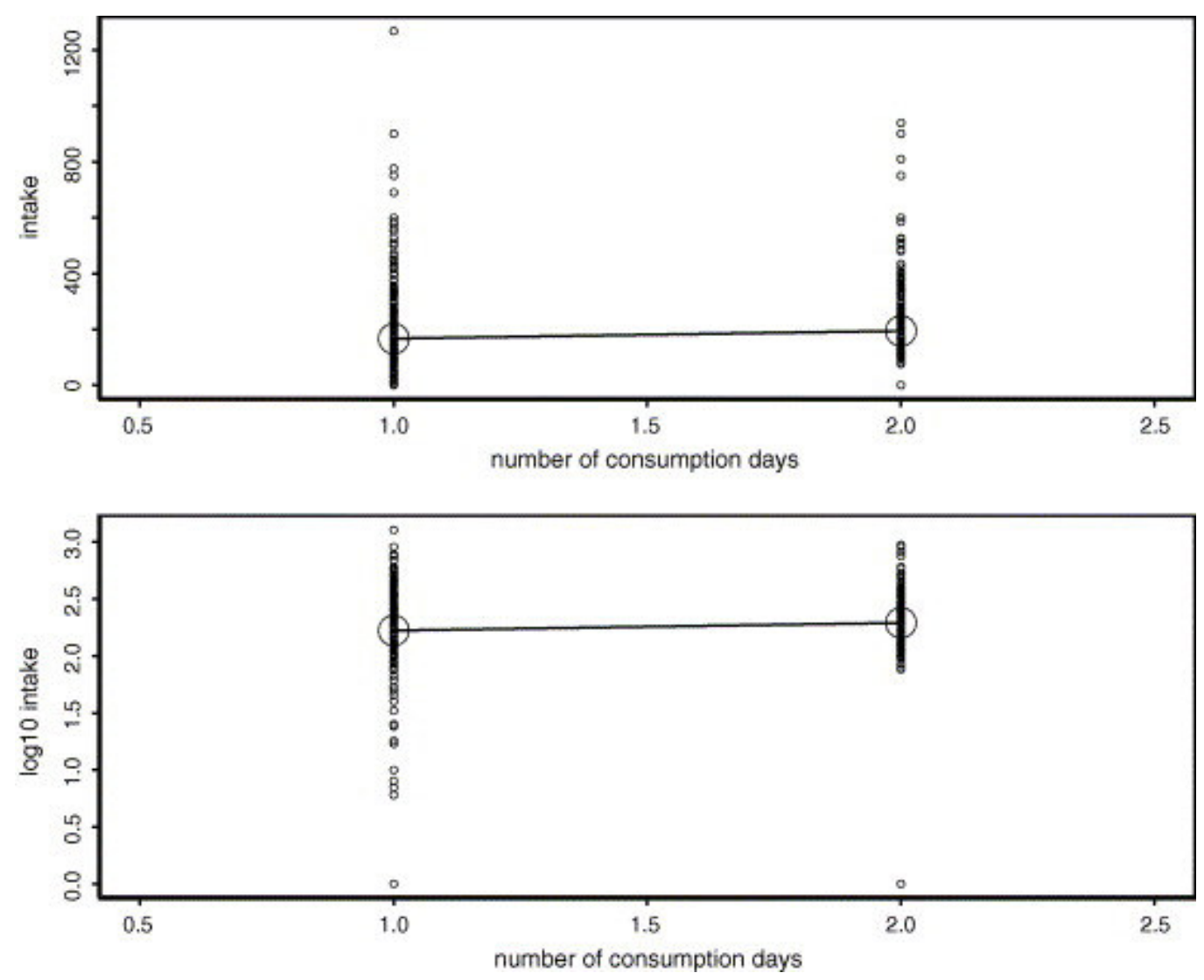

Fig. 5. Observed intake amounts of wine $(\mathrm{ml})$ plotted against number of consumption days (lower panel: intakes on log 10-scale). The large marks in the upper plot indicate the geometric means, in the lower plot the arithmetic mean log-intakes. Ratio of the $\mathrm{GMs}=1.21$. For persons with two consumption days, only one (randomly drawn) amount is plotted.

Fig. 6 shows the amounts of wine (in $\mathrm{ml} /$ day) consumed per day, plotted against age, together with a fitted regression model, expressing the relationship of (geometric) mean intake amounts with age. Fig. 7 shows the same function fitted, but now assuming different values for parameter $a$ ( $=$ the intake at age zero according to the curve) for people that used wine on only one, or on both of the registration days. This results in a significantly better fit (twice the difference in log-likelihoods is Chi-square distributed with one $\mathrm{df}, P=0.005)$, the ratio of the two estimated values for a being 1.16. This is lower than the value of 1.21 estimated earlier with the method in Fig. 5. The model was also re-fit allowing different values for the variances of the regression residuals associated to people with one or two consumption days, but this did not result in a significantly better fit. 


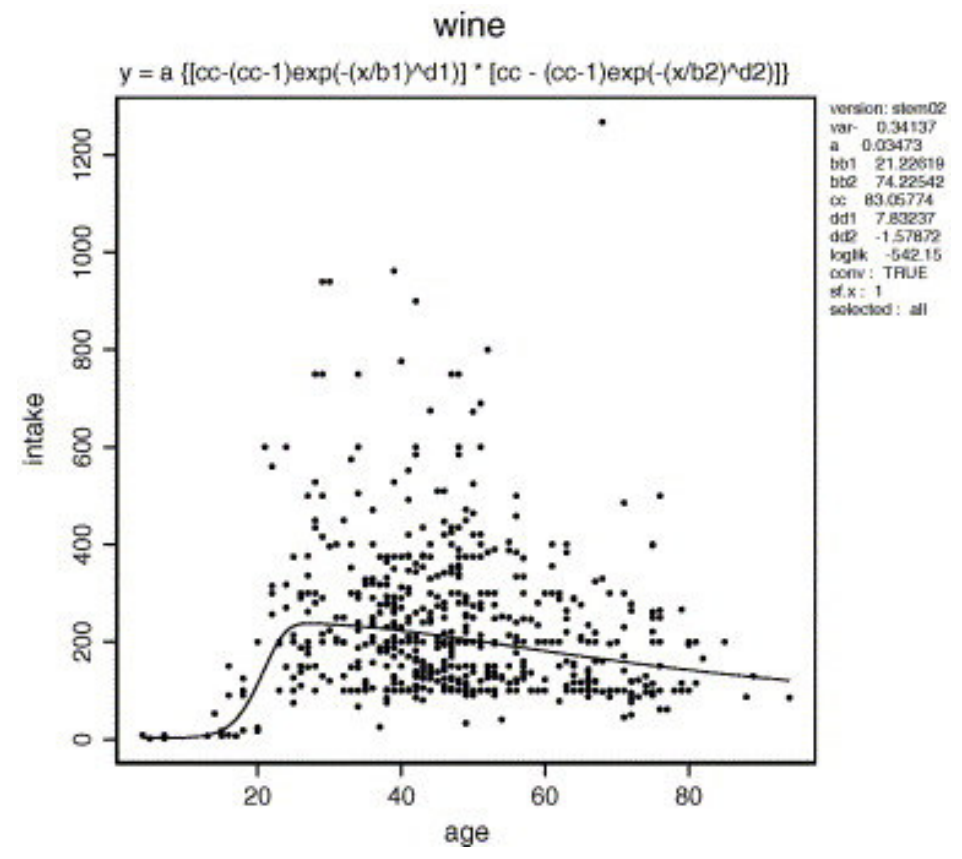

Fig. 6. Observed intakes of wine (in $\mathrm{ml}$ /day) plotted as a function of age, together with a fitted regression function. Note that the data show a preference for particular amounts, such as $100 \mathrm{ml}$.

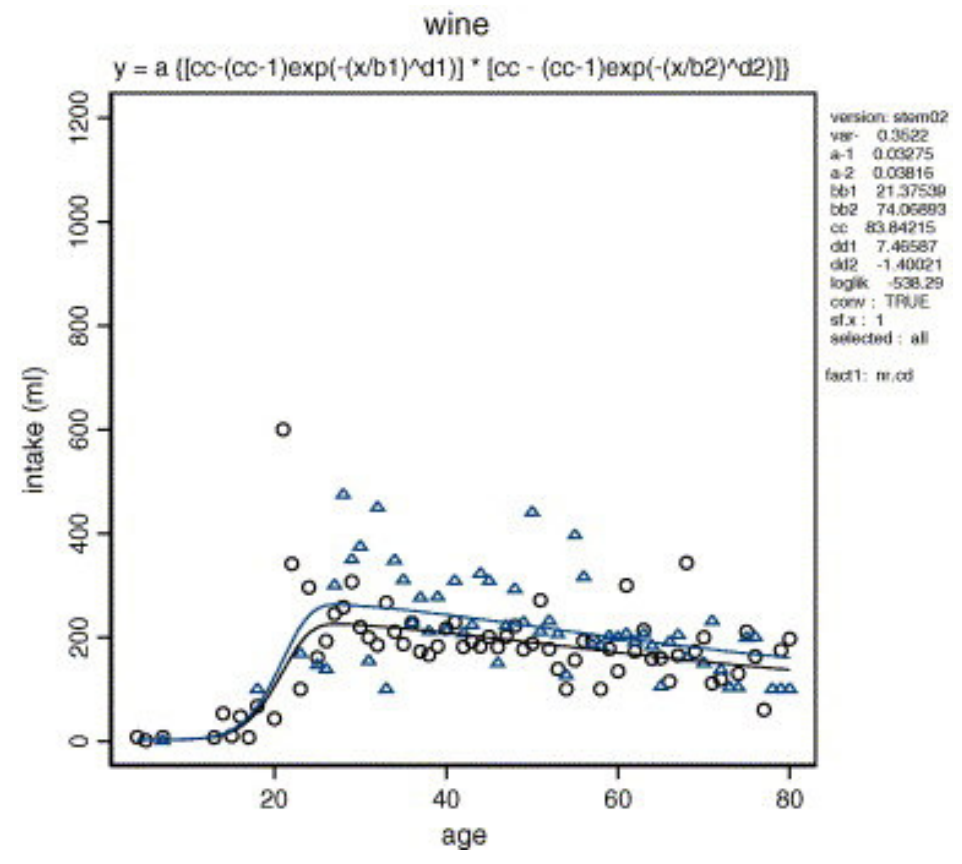

Fig. 7. Observed (geometric) mean intakes of wine (in $\mathrm{ml}$ ) plotted as a function of age, together with a fitted regression function. Here, the parameter $a$ was allowed to be different between persons with one or with two observed consumption days. Although the difference in amounts consumed by less or more frequent wine consumers is statistically significant, the difference is only $15 \%$ (see ratio of $a-1$ and $a-2$ ). 
The fact that the data reveal a significant difference in amounts of wine consumed per day between the persons showing one or two consumption days should be included in reporting the model results, preferably with some indication of the potential error in the final estimates of the model (e.g., by assuming various relationships between frequency and amount that may be possible given the $15 \%$ higher value of $a$ as observed in the analysis of Fig. 7).

In a similar analysis as illustrated in Fig. 7, it was found that males show a significantly higher wine consumption per day than females, the relationship of intake with age otherwise being the same (results not shown). The ratio of the parameter values of a was 1.08 , so the difference between sexes is only around $8 \%$.

In the case of potato, the amounts consumed (per kg body weight per day) showed a simple monotone relationship with age, in a way that is typical for energy intake. The fit of the regression function could be significantly improved by assuming not only parameter $a$ (the intake at age zero according to the fitted curve), but also parameter $b$ (the slope of the curve) being different between sexes (see Fig. 8). Note that these curves are very similar, and yet statistically significantly different, due to the large amount of data in this case.

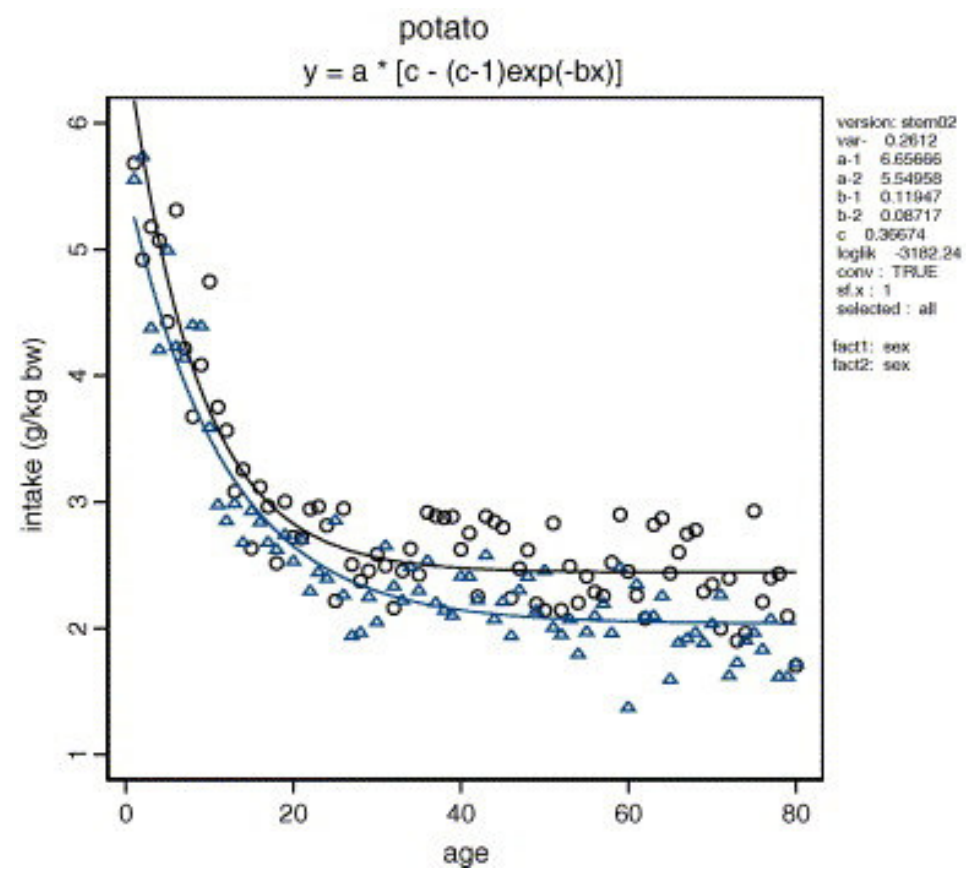

Fig. 8. Observed (geometric) mean intakes of potato ( $\mathrm{g} / \mathrm{kg} \mathrm{BW}$ ) plotted against age (circles for males, triangles for females), with fitted regression model allowing for both parameters $a$ and $b$ being different between sexes. The parameter var denotes the variance of the residuals (on log-scale).

After having decided what regression function to use, and which covariates to include, the regression residuals may be examined for that selected situation. The analyses shown in the examples assumed lognormal residuals, and this can be checked by plotting their distributions, and making QQ-plots (i.e., plots of the observed quantiles against the theoretical quantiles based on the assumed normal distribution). Fig. 9 shows the results for the potato example, where the residuals appear to deviate from the lognormal distribution in the lower tail. 

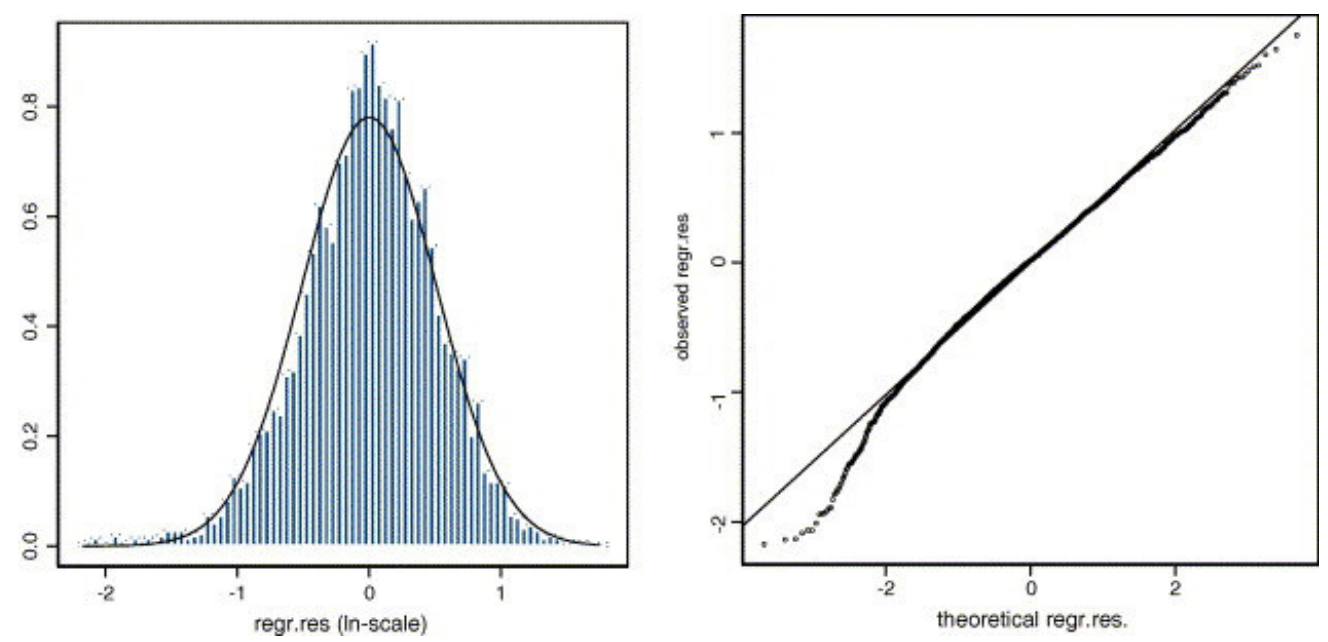

Fig. 9. Distribution of regression residuals for potato intakes (histogram in the left panel, QQ-plot in the right panel). The left tail appears to deviate from the theoretical lognormal distribution (as indicated by the straight line).

\section{Applications of the model in exposure assessment}

In risk assessment of chemicals two questions play a central role: (i) is the longterm exposure to a chemical lower than the chronic exposure limit and (ii) is the short-term exposure (e.g., on a single day) to a chemical lower than the acute exposure limit. For the same chemical the exposure limit for acute exposure (e.g., ARfD = acute reference dose) is always higher than (or equal to) the chronic exposure limit (e.g., ADI, RfD). For some chemicals it may be relevant to do both comparisons, while for others (e.g., compounds that accumulate in the body) only the chronic comparison may be relevant.

It will now be discussed how the model discussed here (STEM.II) can be used for deriving the relevant intake information for both chronic and acute comparisons with exposure limits. In the examples below it is assumed that the food component of interest has a constant concentration. Later on it will be discussed how variation in concentrations may be dealt with in the exposure assessment.

\subsection{Long-term intake and chronic exposure limits}

When comparing a chronic exposure limit with the human intake, a relevant question to be answered is: What fraction of the population has a long-term average intake higher than the exposure limit? For situations where almost every individual has a nonzero intake at almost every day (which occurs when a chemical is present in various basic food products), this can be done by applying earlier published models (e.g., Slob, 1993a and Nusser et al., 1996). These models are not suitable, however, for assessing the distribution of long-term intakes when a chemical occurs in products that are consumed incidentally. In that case, differences in consumption frequencies between individuals must be taken into account. It should be noted however, that chronic exposure limits are typically derived from animal studies where the animals received a constant daily dose over time. The question of what constitutes the comparable long-term exposure in a human being with incidental (peak) intakes is far from trivial. In current practice, and in the absence of a better alternative, the cumulative dose is often taken as the entity to compare different exposure patterns in time. Therefore, the arithmetic mean intake per day is a relevant long-term intake 
measure for an individual person. This is important to note, since the model describes the usual intake as the median daily intake in an individual. When the log-transformation was used in analysing the data, this can be easily corrected for using the relationship (see e.g., Aitchison and Brown, 1966):

\section{$\mathrm{AM}_{\text {ind }} \mid$ consumption day $=M_{\text {ind }} \exp \left(0.5 \sigma_{s}^{2}\right)$,}

where $A M_{\text {ind }}$ denotes the arithmetic mean intake in an individual, and $M_{\text {ind }}$ the median (or geometric mean) intake in an individual (estimated by the regression function of age). To obtain the (marginal) average intake over both consumptionand non-consumption days, this value still needs to be multiplied with $n$, the fraction of non-consumption days:

$$
\mathrm{AM}_{\text {ind }}=\pi M_{\mathrm{ind}} \exp \left(0.5 \sigma_{s}^{2}\right)
$$

Thus, the arithmetic mean intake in individuals is distributed as

$$
\mathrm{AM}_{\text {ind }} \sim \Lambda\left(g(t)+0.5 \sigma_{\mathrm{g}}^{2}+\log (\pi) ; \sigma_{\mathrm{a}}^{2}\right) .
$$

If parameter $n$ were constant among individuals, expression (6) directly defines the probability of exceeding a given limit value, $\lambda$ :

$$
P_{\text {exweding } \lambda}=1-\Phi\left(\frac{\log (\lambda)-g(t)-0.5 \sigma_{s}-\log (\pi)}{\tau_{s}^{2}}\right) .
$$

However, since $n$ varies among individuals itself, this probability must be convoluted with the distribution of $\Pi$ :

$$
P_{\text {exceeding } \lambda}=\int_{\pi} h(\pi)\left[1-\Phi\left(\frac{\log (\lambda)-g(t)-0.5 \sigma_{s}-\log (\pi)}{\sigma_{u}^{2}}\right)\right] \mathrm{d} \pi_{s}
$$

where $h(n)$ denotes the beta density of the consumption frequency. This expression may be evaluated numerically, by subdividing the potential range of $\pi$ , i.e., the interval $(0,1)$, in a sufficient number of bins.

The resulting fraction of the population exceeding the limit $\lambda$ is a function of age, since both consumption frequency $n$ and amount $g(t)$ are functions of age. This fraction therefore relates to the usual intake in the theoretical sense of the expected intake (= arithmetic mean intake over infinite time) at that age in a particular individual. Obviously, within individuals the number of days at a given age cannot be infinite, and therefore the usual intake should be interpreted in the sense of statistical expectation. Further, it should be noted that differences in usual intakes among age groups may be due to age effects and/or to generation effects.

One might also be interested in the (variation in) long-term intake in the sense of the total intake over a whole life span in each individual. For compounds that result in no or very few zero daily intakes, the associated fraction in the population exceeding the exposure limit can be evaluated relatively easily (Slob, 1993b). For incidentally consumed products, however, it seems that this fraction can only be estimated by a Monte Carlo approach, i.e., randomly sampling individuals from both the consumption frequency distribution and the amount distribution. Whatever method is used, an essential assumption to be made in this life span assessment is that individuals show a consumption frequency during their lives that changes with age in parallel to the age related mean consumption frequency (see Fig. 3 or expression (1)). Further, an individual's usual consumption amount must be assumed to change with age in parallel with the 
estimated median amount as a function of age (see Fig. 6 or expression (2)). This may not be realistic, e.g., some individuals may change their consumption pattern systematically at one or various points during their lives (diets). Further, for many food products there may be a generation (cohort) effect that is confounded with the age effect in a single food consumption survey. It might be possible to solve this, by analyzing various consumption surveys that have been performed at different points in time (over a total period of several decades).

\subsubsection{Illustrative example}

In current practice, the fraction of the population exceeding a (chronic) limit value is often estimated by simply taking the fraction of the individuals in the consumption survey data showing an average intake (over the observed days) that is higher than the limit value. We can now examine how this direct method compares with applying the model discussed here. Fig. 10 shows the estimated fractions for the potato data (limit value $=4 \mathrm{~g} / \mathrm{kg} /$ day) based on the model (upper left panel) and based on the direct method (upper right panel). Both methods result in similar values. Obviously, the scatter in the results from the direct method is hard to work with in a risk assessment, but one might consider fitting a regression function to these scattered data. The lower panels of this same figure show the results for a higher limit value (limit value $=15 \mathrm{~g} / \mathrm{kg} / \mathrm{day}$ ). Fig. 11 shows similar results for the wine example. This illustrates that the direct method collapses when the fraction exceeding the limit value becomes too low. Also note that the outcome from the parametric model immediately shows if the probability of exceeding the exposure limit is substantially different among sexes (or other covariates that might have been considered). 
Food and Chemical Toxicology Volume 44, Issue 7 , July 2006, Pages 933-951
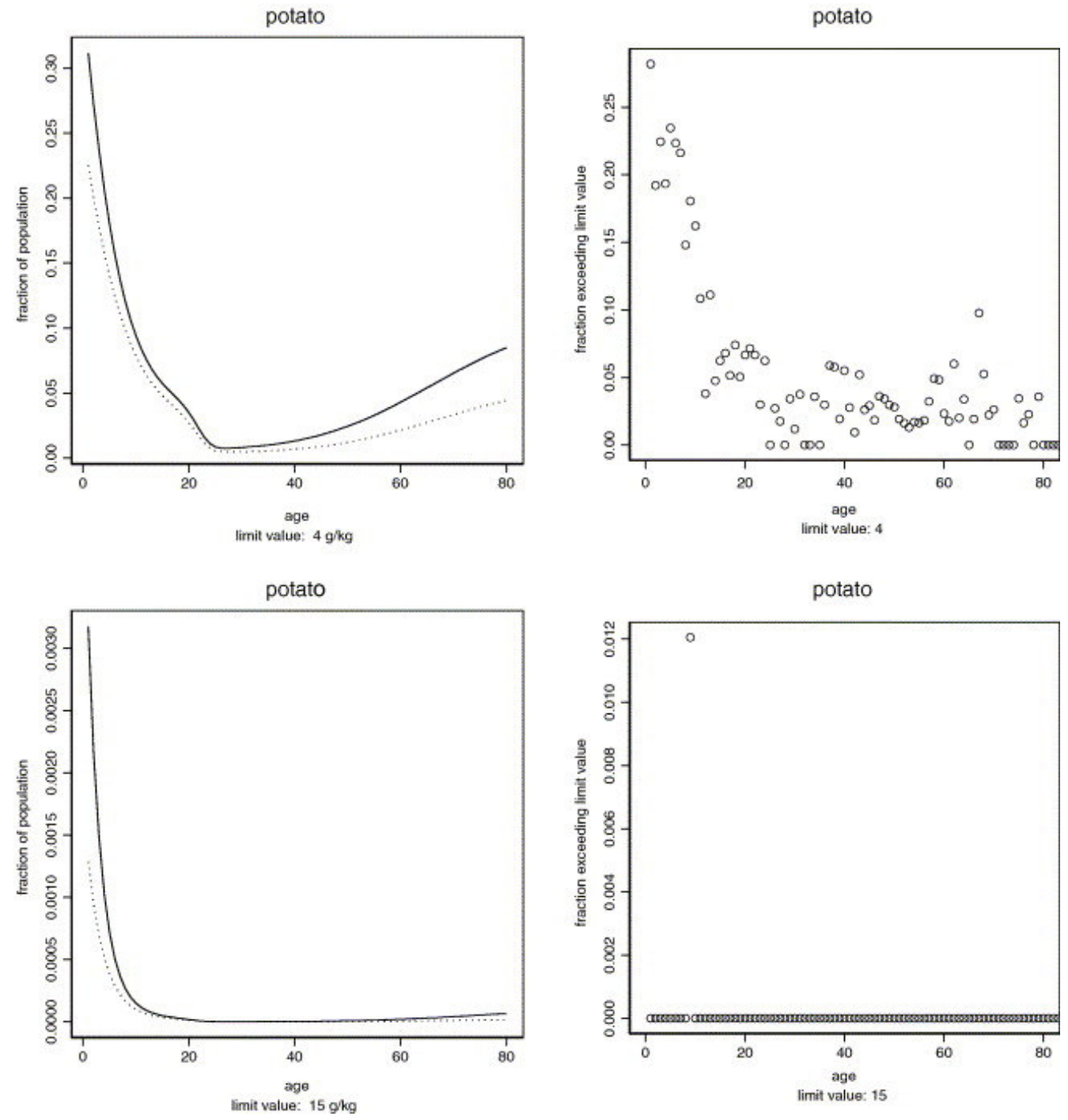

Fig. 10. Age-specific fraction of the population for which the long-term intake of potatoes exceeds a limit value of $4 \mathrm{~g} / \mathrm{kg} /$ day (upper panels) or $15 \mathrm{~g} / \mathrm{kg} / \mathrm{day}$ (lower panels). The left panels show the results based on the model discussed in this paper (dashed line for females), the right panels show the fractions of the observations exceeding the limit value for each age class. 

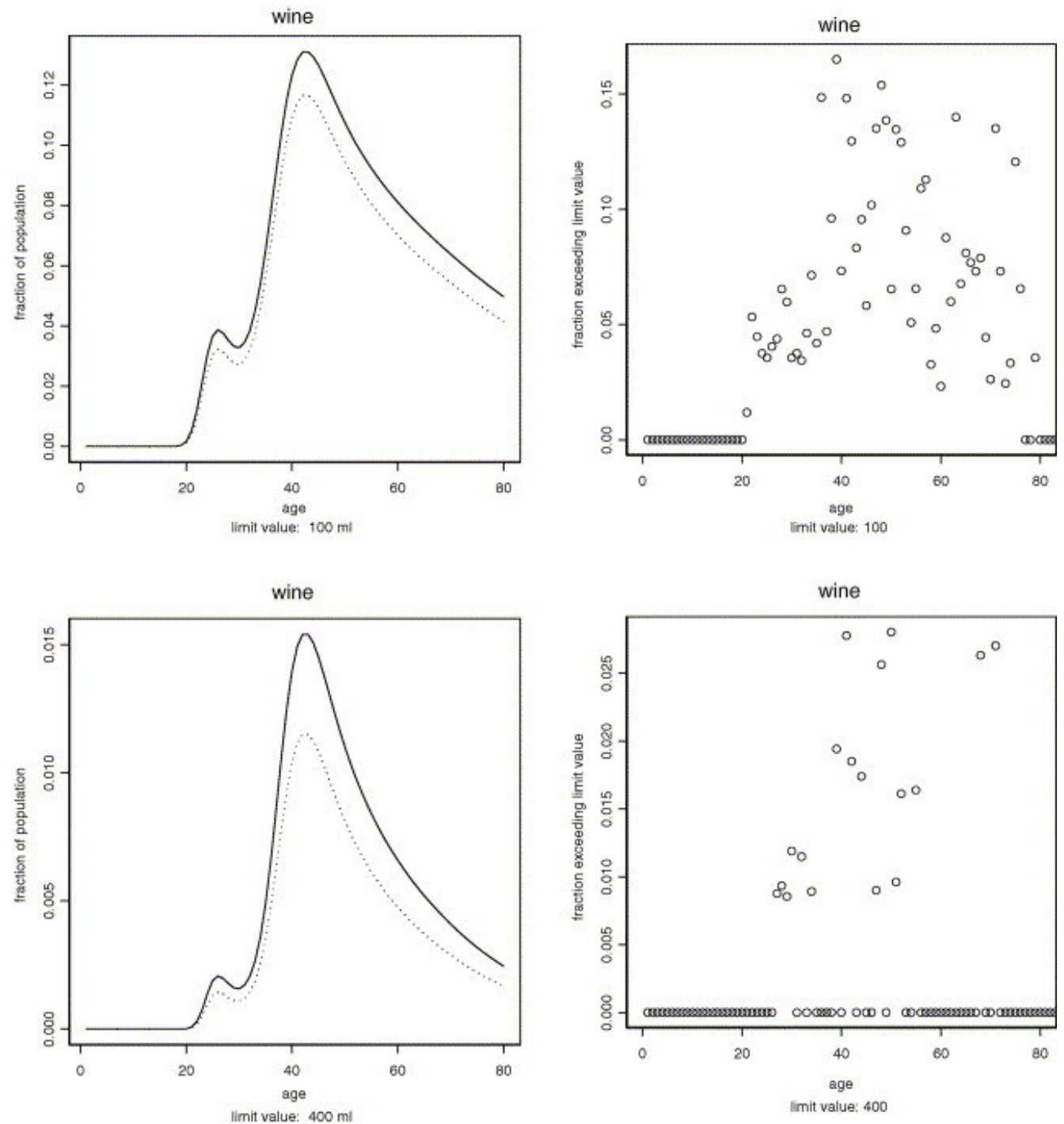

Fig. 11. Age-specific fraction of the population for which the long-term intake of wine exceeds a limit value of $100 \mathrm{ml} /$ day (upper panels) or $400 \mathrm{ml} /$ day (lower panels). The left panels show the results based on the model discussed in this paper (dashed curve for females), the right panels show the fraction of the observations exceeding the limit value.

\subsection{Short-term intake and acute exposure limits}

The probability of exceeding an acute limit value is sometimes assessed by taking the fraction of observed daily intakes exceeding that limit value. Again, this (direct) approach of estimating this probability directly from the observations can be compared with the (indirect) approach of deriving the probability from the model that was fitted to the data. In the case of the acute exposure situation, both approaches give quite different results, not only quantitatively, but also qualitatively. To illustrate this, two food products (potatoes and grapes) are compared by choosing their limit values such that for both products the same probability of exceeding the acute limit is found according to the direct method. Fig. 12 shows the observed fraction of daily intakes exceeding a limit value for potato and grapes, such that the overall fraction (i.e., over age) was 0.014 in both cases. In this example, age was restricted to the range 20-60 years of age, 
and the observed fractions do not appear to depend on age according to these plots, both for potatoes and for grapes (although for grapes one might argue about a positive trend with age).
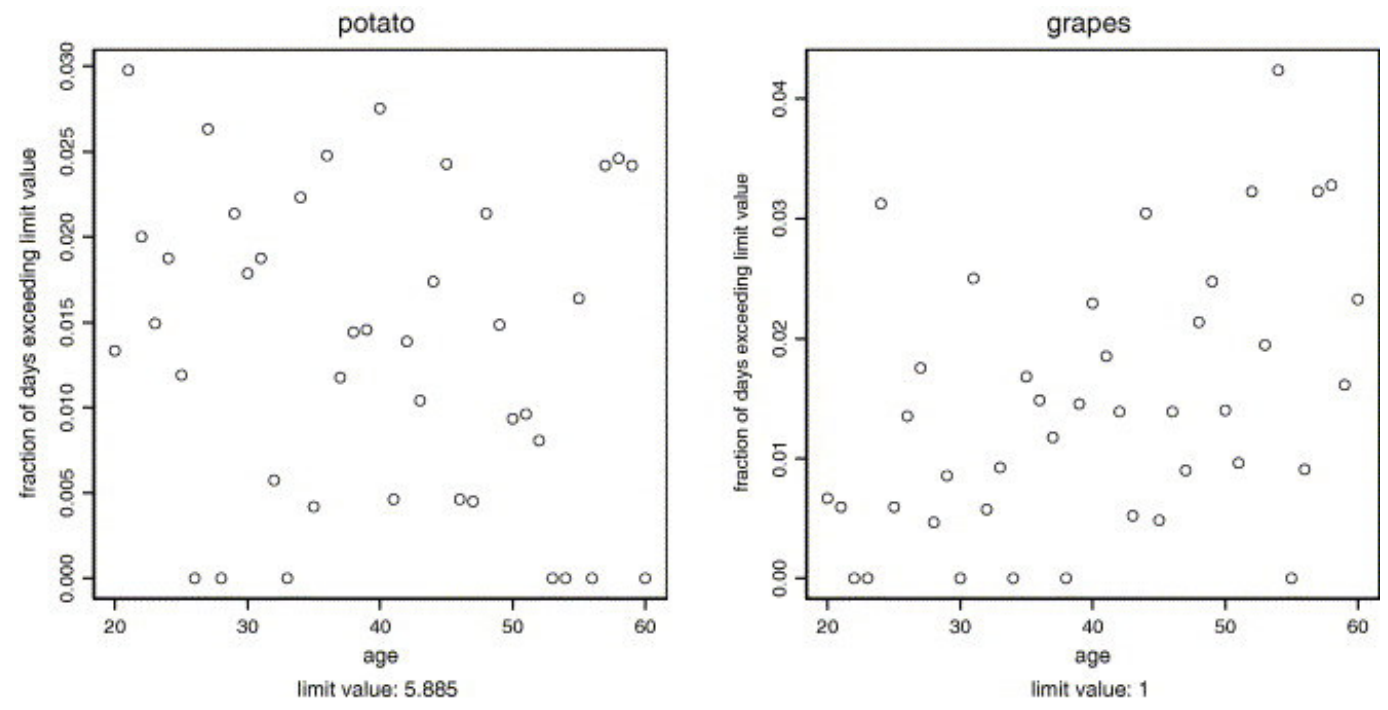

Fig. 12. Observed fractions of the observations (i.e., daily intakes) exceeding an acute limit value for potato and grapes. In both cases the overall (for all ages between 20 and 60 years) fraction exceeding the limit value was 0.014 .

For the same consumption data the indirect method of deriving the probability of exceeding the acute limit value from the fitted model gives a quite different picture. The reason is that the model is able to distinguish between variation among individuals and variation among days (both with respect to amount and frequency of consumption). It follows that for exceeding an acute limit value, the result should include not one, but two probabilities, i.e., it should state how many people may exceed the limit how often. Obviously, a smaller and smaller fraction of the population will exceed the limit value more and more often. This may be represented in a single curve (for a given age group), as illustrated in Fig. 13. As this figure shows, the curves for potato and grapes are quite different, even though the observed fractions estimated by the direct method were similar. This can be explained by the fact that in the case of potato many daily intakes are just below the limit value, while for grapes most intakes are zero, i.e., infinitely far away from the limit value. The direct method does not take this difference into account, and apparently its results are misleading. 

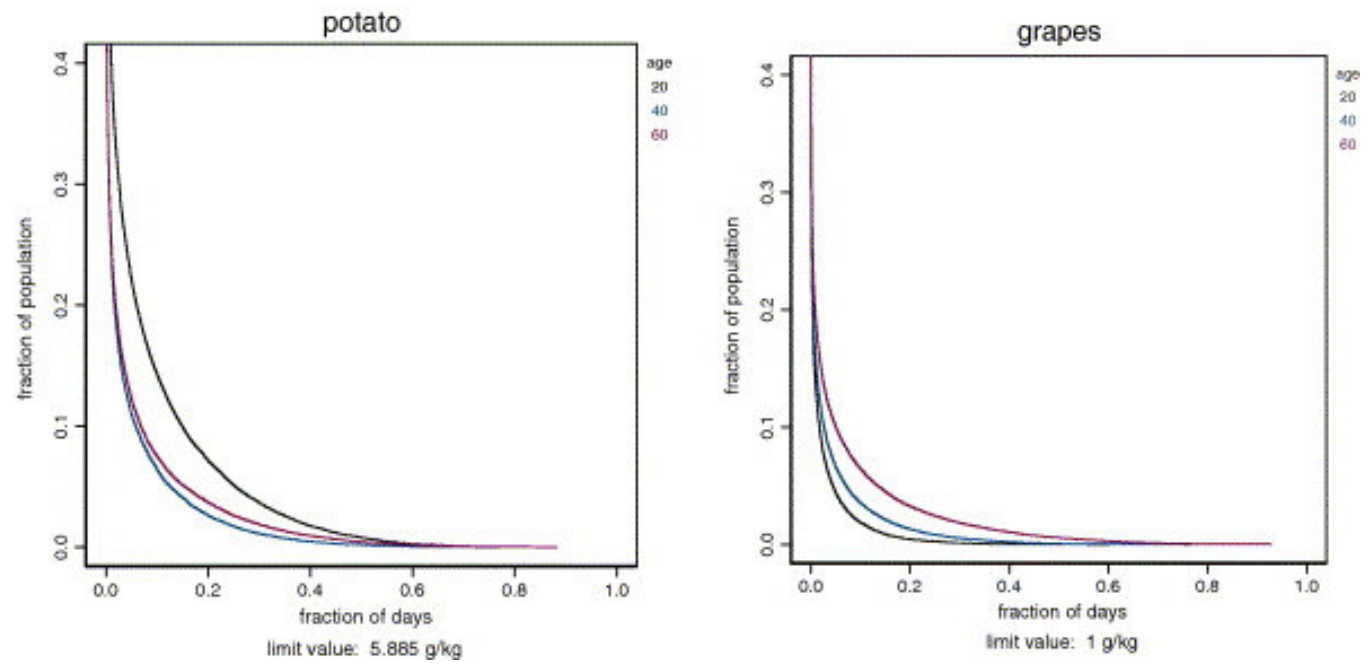

Fig. 13. Fraction of the population ( $y$-axis) against fraction of days ( $x$-axis) at which the limit value is exceeded, for potato and grapes, as estimated by the model fitted to the consumption data. Note that these curves should be read analogous to survival curves, i.e., the fraction of the population on the $y$-axis will exceed the limit value by the associated fraction of days as indicated at the $x$ axis, or by a higher fraction of days. The three curves represent people of 20,40 and 60 years of age.

Another way of presenting the results derived from the model presented in Fig. 13 , is as illustrated in Fig. 14. This figure shows (the lower bound of) the fraction of days for which the acute exposure limit is exceeded in $5 \%$ of the population (as a function of age). Put another way, $95 \%$ of the population would not exceed the limit value more often than the value indicated on the $y$-axis. Also note that Fig. 14 reveals a clear effect of age for both products, while such was not (clearly) apparent from the direct method (Fig. 12). Of course, Fig. 14 could be extended by incorporating other percentiles of the population, to get a more complete picture.
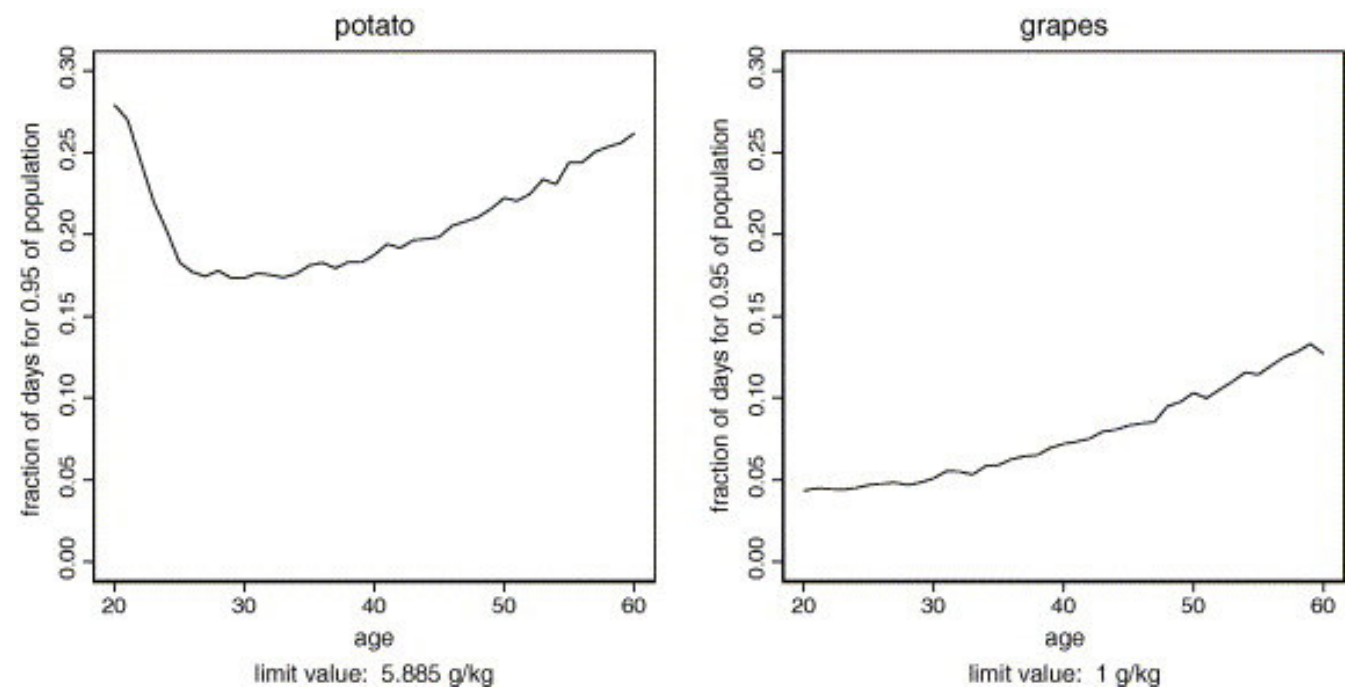

Fig. 14. 95th Percentile of the fraction of days at which the acute exposure limit is exceeded, as a function of age. Thus, $95 \%$ of the human population will exceed 
the limit by a fraction of days as indicated, or lower, while $5 \%$ of the population will exceed the limit at a higher fraction of days.

\section{Discussion}

This paper discussed a statistical model for describing food consumption patterns in the human population, focusing on incidentally consumed products. One of its applications is to assess whether or not the intake of food components by humans comply with certain intake standards or limit values. In our examples we used limit values that should not be exceeded, but minimum requirement values (e.g., essential nutrients) can be equally dealt with. The probability of exceeding a limit value is estimated from the statistical model, and therefore may be considered as an indirect (two-step) method, as opposed to the direct method where this probability is assessed directly as the observed fraction of calculated intakes exceeding the limit value (i.e., one step).

As shown by the examples (Fig. 10 and Fig. 11), the direct method may give reasonable results when the average of the observed intakes in each individual is compared to a (chronic) limit value, but only when the fraction exceeding the limit value is not too low. For lower fractions (relatively high limit values) the direct method fails, simply because of the fact that the observed fractions will tend to be zero. Another problem of the direct method is that as soon as all observed nonzero intakes are above the limit value, the observed fraction will remain the same when the limit value is reduced to whatever lower value. Obviously, this does not reflect reality. While the first problem could be dealt with by Monte Carlo sampling (by iterative re-sampling with replacement), the second problem will remain.

In comparing acute (daily) intakes with acute limit values the inadequacy of the direct approach becomes much more apparent. The fraction of the observed daily intakes that exceed an acute limit value does not really have a useful interpretation to begin with. For instance, the situation where $5 \%$ of the population exceeds the limit value every day cannot be distinguished from the situation where every individual in the population exceeds the limit value in $5 \%$ of the days. Recently published methods of probabilistic dietary intake assessment by Monte Carlo techniques (Gibney and van der Voet, 2003 and Van der Voet et al., 2004) suffer from the same problem: the fraction of the resulting intake distributions exceeding some limit value reflects person-days, not individuals.

Current debates of taking the whole population or only the "consumers" into account further illustrates the conceptual problem here: the binary distinction consumers vs. non-consumers is based on the fact that the data consist of zeros and non-zeros, which does not imply that this distinction exists in the human population. When a consumer is defined as an individual in the survey with at least one nonzero consumption day, then the number of consumers is almost completely determined by the number of registration days per individual (Lambe et al., 2000), i.e., it is a property of the data rather than of the human population.

The issue of consumers vs. non-consumers thus appoints the fundamental objection against the direct approach: it sticks to the level of the data, and therefore the results of the analysis are, strictly speaking, a statement about the properties of the data. We are, however, not interested in the data themselves, but rather in reality behind the data. Therefore, the data first need to be lifted to the level of interest, in the present case the behavior of the whole population, during a much longer period of time, acknowledging the complex structure of 
consumption patterns in the human population. The food consumption data are subject to various gaps in the information they provide, and these need to be filled to get a complete picture of reality. This can only be done by making assumptions, and the parametric model discussed here contains quite some of them. The parametric model and the direct method may be regarded as two extreme approaches, with various options in between. The method proposed by Nusser et al. (1996) is an example of an intermediate approach. For instance, they do use the normal distribution, but make various corrections that strongly hinge on the particular dataset. As another example, Dodd (1996) describes a method for estimating the consumption frequency distribution without assuming a particular type of distribution (such as the beta-distribution, or any other).

These intermediate approaches of modelling the intake data appear quite reasonable at first sight. After all, a model that hinges less on assumptions and more on the data at hand can only be better. Yet, there are other considerations that need to be taken into account as well. A general point is that emphasis on one aspect of a problem usually goes at the expense of other aspects. Thus, a statistical analysis that puts much effort in getting the distribution "right" might overlook other important aspects, such as the relationship of consumption behavior with age, or differences between sexes or other subgroups or covariates (e.g., illness). Further, it should be noted that food consumption data contain all sorts of irregularities or even errors. For instance, underreporting is a well-known phenomenon in food surveys. As another example, amounts are usually not measured but roughly estimated by the subjects in the survey using example pictures. Many other sources of errors may be thought of. Therefore, a very strong focus on the distribution of the observations does not appear a first priority: the resulting estimates of the percentiles will to some extent be inaccurate anyway, due to the data being imperfect by themselves.

It seems a better strategy to first of all fully analyse the consumption data with a parametric model as discussed here, to get a complete picture of the consumption patterns in the population, including the impact of potential covariates such as sex, social class, or illness. Such knowledge is often highly relevant by itself. And, secondary, ignoring relevant covariates may distort the distribution of the residuals, and correcting these distortions by some transformation or smoothing technique is not appropriate. For instance, the distribution of the total population will usually be skewed (on log-scale) due to the fact that children have different intakes from adults. Taking age into account in the model is a better strategy than making the tail disappear by some transformation.

Therefore, it is suggested to first of all model the consumption data to get a complete picture, using a parametric model based on (default) assumptions, and only evaluate the assumptions afterwards, by examining the model residuals. If the residuals clearly deviate from the default assumptions, one may decide to apply additional techniques, such as Box-Cox transformation, or distribution smoothing techniques. But this should always be done with caution. For instance, the residuals for the potato example (Fig. 9) do not completely comply with the lognormal distribution, in particular the left tail deviates from the lognormal. Here, a Box-Cox transformation might improve the overall agreement to a lognormal distribution, in particular regarding the left tail, but it might at the same time deteriorate rather than improve the description of the right tail. So, if the interest is in the right tail only, it may in some cases be better to maintain the log-transformation. When, on the other hand, interest is in the left tail, one may also put the question if this deviation in the left tail has a relevant cause. Indeed, when those subjects in the consumption survey that reported to be on diet, or to be (temporarily) ill on the registration day, were left out, the deviation 
in the left tail became much less apparent. And when only males from 30 to 60 years of age were considered, the residuals complied nicely with the lognormal distribution (see Fig. 15), after removing three (low) outliers, detected by Grubb's test (Grubb and Beck, 1972). These findings indicate that there might be yet another factor that underlies the observed left tail's deviation. In this particular case, there was a decreasing trend of the variance of the regression residuals with age, and it would be more appropriate to include an age-dependent variance in the model than smoothing the distribution by some transformation.
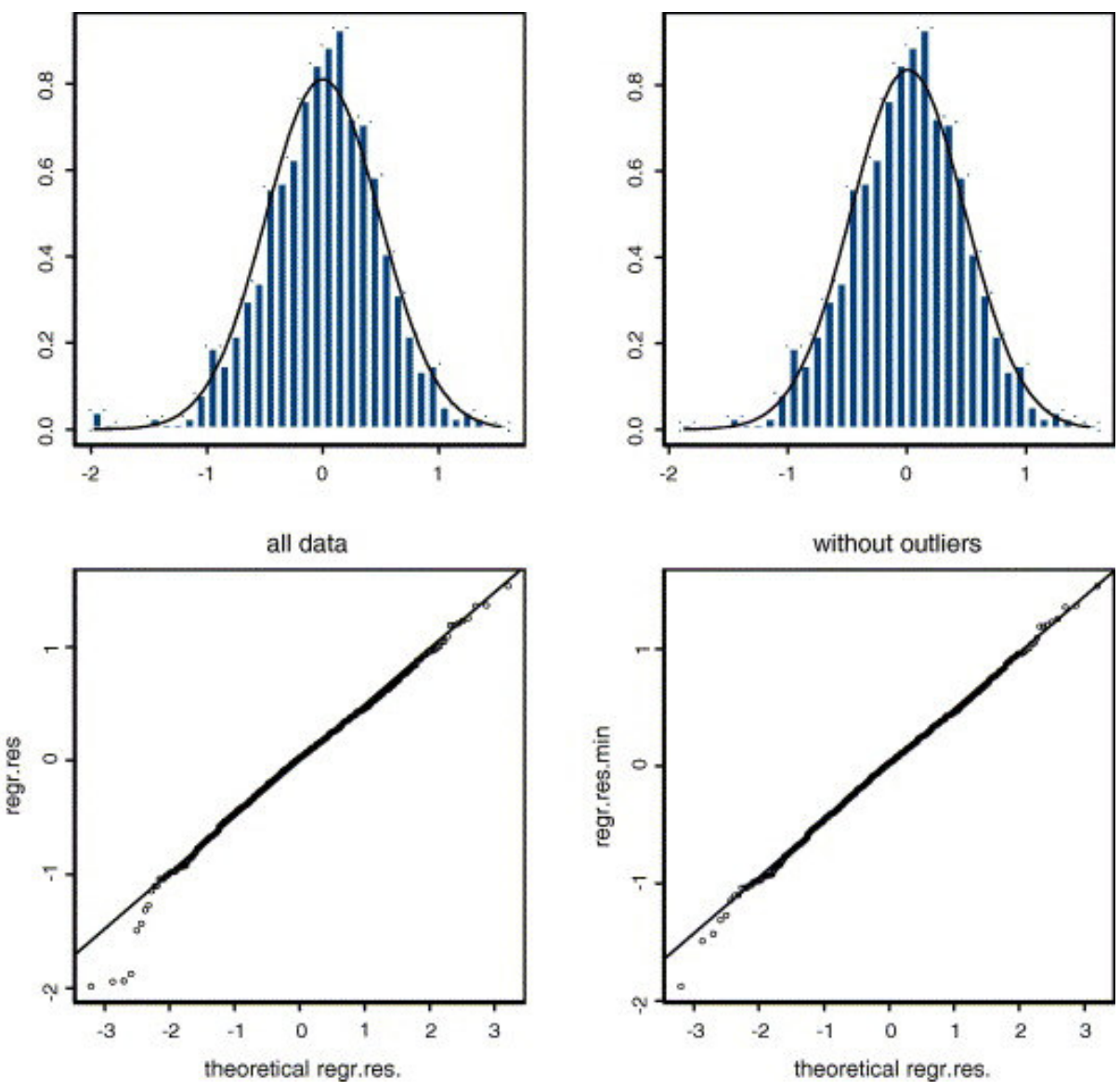

Fig. 15. Regression residuals for potato, for males between 30 and 60 year of age, persons on a diet or ill on that day excluded. In the right panels, three (low) outliers have been removed, which were detected as outliers by Grubb's test $(a=0.05)$.

But in other cases the residuals may without doubt be far from lognormal. In some cases this is explainable. For instance, the amounts of pears consumed per day are typically close to the weight of a single pear, or a multiple of that (people may eat more than one pear, or share one with someone else). Besides that, the respondents in the survey normally do not measure the amount of the products they consume (e.g., they use standard amounts from lists provided by the investigators), and data relating to foods such as pears contain many ties. In such cases, additional techniques as described by Nusser et al. (1996) may be needed. One should realise, however, that this may make the analysis quite complicated. For instance, after having chosen another transformation than the logarithmic, the model should be refitted on that new scale. After that, the residuals may call for yet another transformation, etc. An additional complication 
is that the estimation of the usual (long-term) intake distribution, as described above, makes use of a relationship between the geometric and the arithmetic mean that holds for the lognormal distribution (see expression (5)), but not for other transformations. Of course, in an exposure assessment of a chemical that occurs in tow (or more) foods, their contributions to the total intake being highly different, the resulting distribution of intake amounts may tend to be bi- (or multi-)modal.

The novel aspect of the model presented here concerns the analysis of the consumption frequencies. In the approach suggested, the assumption of the betadistribution is crucial, and needs further validation. Future consumption surveys in the Netherlands intend to ask the respondents for estimating their consumption frequency as accurately as possible for several food products. This information might be useful for validating the assumed beta-distribution. In addition, it might be explored to what extent estimates from the model will differ when assuming another distribution for consumption frequency. Others (Dodd, 1996) have described a method for estimating the distribution of consumption frequencies, without assuming a particular type of distribution. However, the performance of this distribution-free method has (to this author's knowledge) not been investigated by computer simulations, in a way as was done in this paper. It might be that it does not perform as well, especially in the situation of only two registration days per individual. Also, it is not clear how this method could be applied such that the relationship of food frequency with age can be quantified simultaneously.

The most significant consequence of the model discussed here is related to the assessment of exceeding an acute limit value by the human population. While the direct method of counting the fraction of observed daily intakes higher than the limit value results in just a single fraction, the statistical model gives an answer that contains two fractions, i.e., it gives an estimate of the fraction of the population in relation to the fraction of days that the limit value is exceeded. The latter answer is in fact better interpretable, as it better reflects the real life situation. After all, it is evident that some people will exceed the acute limit value more often than others. This more realistic answer implies that for the purpose of risk management both these fractions need to be set at a desirable or acceptable level. The first fraction (of people) is primarily a risk manager's issue, while the second fraction (of days) has a toxicological aspect. The question here is: How often might the acute limit value be exceeded before adverse effects are to be expected? In many cases current toxicological knowledge may be insufficient to give a clear-cut answer to that question, but this might improve in the future, if one is willing to adjust study designs such that they provide information to such questions.

\section{Consequences for probabilistic exposure assessments}

The analyses discussed in this paper showed that assessing the fraction of intakes exceeding an exposure limit by the direct method (i.e., of counting the fraction of observed intakes exceeding the limit value) may not be appropriate, in particular for acute exposure comparisons. The reason is that the daily intakes that are estimated on the basis of food consumption surveys (in combination with the concentrations in the foods) do not comprise a single one-dimensional sample. The variation in the observed intakes results from different sorts of underlying sources of variation, such as variation between individuals in consumption frequency, variation between individuals in consumption amount and variation in consumption amounts within the same individuals. Further, various covariates, such as age and sex, may be involved. While food consumption surveys usually 
comprise a large number of individuals, the information provided for each single individual is very limited. Therefore, our knowledge on food consumption in single individuals will always be poor. The solution provided by statistical modelling is to forget about individuals, but estimate the variation between individuals, or the distribution of the population. Here a statistical model (i.e., a set of assumptions) is needed, to cover the complex structure of the intake data. The direct method of working with the observed intakes as a single distribution does not cover the complex structure of the intake data, and therefore it is inadequate. One might argue that the structure of the data set could be represented in a Monte Carlo sampling scheme, by sampling between and within individuals, but the number of data points within individuals is simply too limited for such an approach.

Various authors (e.g., Slob, 1993a and Nusser et al., 1996) have already emphasised that the distribution of observed average intakes for each individual overestimates the distribution of usual intakes among individuals, unless the total observed variation is corrected for the within person variation. This can only be done by parametric modelling. Parametric modeling is also called for in the situation where the exposure assessment aims to compare acute intakes with an acute exposure limit. In that case, the direct method estimates a fraction of person-days, while actually two fractions are at stake: a fraction of the population, and a fraction of days. Apart from this conceptual limitation, the direct method was shown not to discriminate between two totally different consumption patterns (see Fig. 12), while the indirect method of first describing the intake observations by the parametric model (STEM.II) did result in a substantially different outcome for these two food products.

The main conclusion therefore is that a probabilistic dietary exposure assessment should not be based on the consumption data itself, but on a (parametric) statistical model describing the observed intakes. Depending on the situation, Monte Carlo methods may be additionally needed or not. The various possibilities will now be briefly summarised.

\subsection{Chronic assessments}

When the aim of the assessment is to compare usual intakes with a chronic exposure limit, one may decide to work with the average observed concentration(s) in the relevant food product(s). In that case Monte Carlo is not needed at all. When the chemical at hand occurs in various basic food products, resulting in a nonzero intake in each individual on virtually all days, the model STEM (Slob, 1993a) or a similar model (e.g., Nusser et al., 1996) may be applied to the calculated daily intakes for each individual, based on the average concentrations in the relevant foods. When the interest is in a single food product, resulting in zero intakes for a fraction of the data, the model STEM.II may be used, as illustrated above. Finally, when the chemical occurs in various products that are all consumed incidentally, so that zero daily intakes do occur, STEM.II might be applicable as well. In that case, the daily intakes in each individual may be calculated as the total intake from the various relevant foods, and analyse these intakes by STEM.II. In that case consumption frequency does not relate to a single food, and might be better called intake frequency. Problems may however arise in such an analysis regarding the distribution of amounts, especially when the potential intakes from different food products are very different. Experience with the latter type of analysis is still lacking. 


\subsection{Acute assessments}

For acute intake assessments the variation in the concentrations in food products needs to be taken into account. First consider the situation when only one food product is involved in the assessment. When the concentrations can be assumed to be close to a lognormal distribution, the estimated variance of the logconcentrations can simply be added to the variance between days in logconsumption amounts $\left(\sigma_{\tilde{s}}^{2}\right)$ in STEM.II, and a complete acute exposure assessment can thus be done without Monte Carlo. When the concentrations do not allow for a lognormal distribution, e.g., due to a fairly large fraction of zero concentrations, Monte Carlo methods may be required. In that case, the Monte Carlo concentrations should be combined with Monte Carlo samples from a (parametric) statistical model of consumption, such as STEM.II.

The situation where the interest is in acute intakes due to chemicals occurring in various food products is more complex, and a satisfactory analysis does not seem to be available yet. Of course, STEM.II could be fitted to the consumption data of the various food products of interest separately, but the results could not be simply combined for these food products: the consumption frequencies of different foods as well as their consumption amounts may be correlated, both among persons and within persons on single consumption days. A next step in dietary exposure assessment will be to solve this problem. As long as this problem has not been solved, there appears no other way than to estimate a distribution of person-days in the case of acute exposure via multiple foods. This may be achieved by generating a dataset of total daily intakes by Monte Carlo sampling (from both concentration and consumption data). But even then it might be useful to apply the (partly adjusted) parametric model discussed here to the (large) Monte Carlo sample. Whether such an approach would be better than the direct Monte Carlo approach for this situation needs further investigation.

The main conclusion is that exposure assessments that are fully based on Monte Carlo analysis may not be adequate. Incorporating the parametric model for describing the consumption data rather is particularly relevant for acute exposure assessments. While the Monte Carlo approach results in a distribution of intakes that can only estimate the fraction of person-days exceeding the ARfD, the parametric approach estimates how many people exceed the ARfD how often. The latter type of answer is more informative and relevant for risk assessment. Therefore, a two-step procedure is suggested for acute exposure assessment, where Monte Carlo samples are drawn from the statistical model describing the consumption patterns in the population, instead of sampling from the intake data directly.

The parametric approach has some other advantages. It gives a better view of the relation of intakes with age, as well as with other covariates, and it may reveal outliers, or other peculiarities and errors in the data, which normally remain hidden in a full Monte Carlo approach. Unfortunately, the model discussed here does, as yet, not apply to acute exposure assessment for situations of intakes resulting from multiple foods. In those cases a Monte Carlo approach appears to be the only option, but here one should always keep in mind that the resulting fractions above an exposure limit relate to person-days, not to fractions of individuals in the human population. 


\section{Acknowledgements}

This research was sponsored by the Food and Consumer Product Safety Authority of the Netherlands (VWA), as well as by EU contract Food-CT-2004-506446 SAFE FOODS.

\section{References}

Anon., 1998 Anon, 1998. Zo eet Nederland. Resultaten van de Voedselconsumptie-peiling 1997-1998. Voedingscentrum, Den Haag.

Aitchison and Brown, 1966 J. Aitchison and J.A.C. Brown, The Lognormal Distribution, University Press, Cambridge (1966).

Dodd, 1996 Dodd, K.W., 1996. A technical guide to C-SIDE. Software for intake distribution estimation. Technical Report 96-TR 32. Iowa State University.

Gibney and van der Voet, 2003 M.J. Gibney and H. van der Voet, Introduction to the Monte Carlo project and the approach to the validation of probabilistic models of dietary exposure to selected food chemicals, Food Additives and Contaminants 20 (2003) (suppl. 1), pp. S1-S7. Abstract-MEDLINE । Order Document | Abstract + References in Scopus | Cited By in Scopus

Grubb and Beck, 1972 F.E. Grubb and G. Beck, Extension of sample sizes and percentage points of significance tests of outlying observations, Technometrics 14 (1972), pp. 849-854.

Hertzberg, 1989 R.C. Hertzberg, Fitting a model to categorical response data with application to species extrapolation of toxicity, Health Physics 57 (1989), pp. 405-409. Abstract-EMBASE | AbstractMEDLINE | Order Document | Abstract + References in Scopus | Cited By in Scopus

Lambe et al., 2000 J. Lambe, J. Kearney, C. Leclerq, H.F.J. Zunft, S. De Henauw, C.J.E. LambergAllardt, A. Dunne and M.J. Gibney, The influence of survey duration on estimates of food intakes and its relevance for public health nutrition and food safety issues, European Journal of Clinical Nutrition 54 (2000), pp. 166-173. Abstract-Elsevier BIOBASE | Abstract-MEDLINE | Abstract-EMBASE | Order Document | Full Text via CrossRef | Abstract + References in Scopus | Cited By in Scopus

Nusser et al., 1996 S.M. Nusser, A.L. Carriquiry, K.W. Dodd and W.A. Fuller, A semiparametric transformation approach to estimating usual daily intake distributions, JASA 91 (1996), pp. 14401449. Abstract-EconLit | Order Document | Abstract + References in Scopus | Cited By in Scopus

Slob, 1993a W. Slob, Modeling long-term exposure of the whole population to chemicals in food, Risk Analysis 13 (1993), pp. 525-530. Abstract-MEDLINE | Abstract-EMBASE | Order Document | Full Text via CrossRef | Abstract + References in Scopus | Cited By in Scopus

Slob, 1993b Slob, W., 1993b. Modelling human exposure to chemicals in food. RIVM, Report No 639102002, April 1993.

Slob, 2002 W. Slob, Dose-response modeling of continuous endpoints, Toxicological Sciences 66 (2002), pp. 298-312. Abstract-EMBASE | Abstract-MEDLINE | Abstract-Elsevier BIOBASE | Order Document | Full Text via CrossRef | Abstract + References in Scopus | Cited By in Scopus

Teunis and Slob, 1999 P.F.M. Teunis and W. Slob, The statistical analysis of fractions resulting from microbial counts, Quantitative Microbiology 1 (1999), pp. 63-88. Full Text via CrossRef

Van der Voet et al., 2004 Van der Voet, H., de Boer, W.J., Boon, P.E., van Donkersgoed, G., van Klaveren, J.D., 2004. MCRA Release 3 Reference Guide. Report Biometris and RIKILT, Wageningen. Available from: <http://mcra.rikilt.wur.nl>. 